Elsevier required licence: (c) $<2015>$. This manuscript version is made available under the CC-BY-NC-ND 4.0 license http://creativecommons.org/licenses/bync-nd/4.0/ 


\title{
Connecting the dots: econometric methods for uncovering networks with an application to the Australian financial institutions*
}

\author{
Mikhail Anufriev \\ University of Technology Sydney, Business School, Economics Discipline Group \\ Sydney, NSW 2001, Australia \\ Valentyn Panchenko** \\ University of New South Wales, UNSW Business School, Economics \\ Sydney, NSW 2052, Australia
}

15 July 2015

\begin{abstract}
This paper connects variance-covariance estimation methods, Gaussian graphical models, and the growing literature on economic and financial networks. We construct the network using the concept of partial correlations which captures direct linear dependence between any two entities, conditional on dependence between all other entities. We relate the centrality measures of this network to shock propagation. The methodology is applied to construct the perceived network of publicly traded Australian banks and their connections to domestic economic sectors and international markets. We find strong links between the big four Australian banks, real estate and other sectors of the economy, and determine which entities play a central role in transmitting and absorbing the shocks.
\end{abstract}

${ }^{*}$ We benefited from helpful comments by Shu-Heng Chen, Mardi Dungey, Hakan Eratalay and Matt Jackson, two anonymous referees, as well as by participants of the 1st Conference on Recent Developments in Financial Econometrics and Applications, Deakin University; the Summer Workshop at the Centre for Mathematical Social Sciences, the University of Auckland; the Economics and Financial Markets Workshop, the University of Technology, Sydney; and seminars at the University of Sydney and the European University at St. Petersburg. This research was supported by the Centre for International Finance and Regulation (project number E233), which is funded by the Commonwealth and NSW Governments and supported by other Consortium members (see www.cifr.edu.au).

${ }^{* *}$ Corresponding author. Telephone: +61 2 93853363; E-mail: v.panchenko@unsw.edu.au. 


\section{Introduction}

The global financial crisis has called for a better understanding of the vulnerabilities and risks of financial markets. Connections between different segments of the market play an important role in determining the extent and patterns of these risks. These interconnections can be studied using the tools of network theory. Network modeling is a novel and rapidly developing field in social sciences, economics, and finance (see e.g., Jackson, 2008; Allen and Babus, 2009).

This paper brings together ideas from network theory, the financial econometric literature on variance-covariance modeling, and statistical literature on Gaussian graphical models (GGM). The GGM are widely used for the reconstruction of networks when the actual network structure is unobservable. A prominent example of this use is the biological literature on networks of genes, proteins, etc. (e.g., Rice et al., 2005). These methods are relatively new in economics and finance. We apply the GGM to reconstruct the network of partial correlations between different Australian banks, domestic economic sectors and, international markets. We use network theory to study this network and interpret its properties.

GGM are developed to visualize the conditional dependencies between different elements of a multivariate random variable through a graph of partial correlations (see Whittaker, 2009, for detailed treatment). Partial correlations capture bi-variate linear dependencies between any two elements of the random variable, conditional on a set of all remaining elements. As we show, this feature is useful to separate a direct dependence, between a pair of economic sectors or entities, from indirect effects coming through the remaining part of the network. For this reason, the partial correlations are well suited for network representation. The standard GGM literature focuses on the reduction of complexity of the conditioning set from the constructed graph (so-called Markov properties). However, our primary focus is on the adaptation and interpretation of popular network-based measures in the context of the graph of partial correlations.

Recent economic literature provides many examples of how financial data can be described from the network perspective. ${ }^{1}$ The studies illustrate the complexity of relationships between financial entities and discover certain network properties which may be

\footnotetext{
${ }^{1}$ See Iori et al. (2008), who study the interbank overnight market; Vitali et al. (2011), who analyze the ownership of transnational corporations; and Sokolov et al. (2012), who investigate Australian interbank transactions, among many others.
} 
important for the aggregate properties of the financial system. An intuitive, but not always formalized, idea is that interdependencies between the entities represent a channel of transmission of a shock. Thus, discovering these interdependencies with the network approach is useful for studying systemic risk, see review of early contributions in Chinazzi and Fagiolo (2013) and recent studies of Acemoglu et al. (2015), Glasserman and Young (2015) and Elliott et al. (2014). Battiston et al. (2012) introduce the DebtRank which is an example of a centrality measure of nodes within the network of financial entities. High centrality of a node would reflect an importance of the node in the shock transmission.

This paper is closely related to the recent work on reconstructing networks from timeseries data by Billio et al. (2012), Dungey et al. (2013), Barigozzi and Brownlees (2013), Diebold and Yilmaz (2014), and, in the Australian context, Dungey et al. (2015). The distinguishing feature of our study is that we are establishing the links between the statistical concepts of correlations, partial correlations, principal components and various centrality measures from the network theory. Moreover, this is the first work mapping the network of perceived financial dependencies between Australian banks, other domestic sectors, and international markets. We use publicly available information on the share prices and indices of the corresponding entities to reconstruct the network of partial correlations between their returns. The returns generally represent market-perceived changes in the value of these entities. The reconstructed networks may be a useful tool for better understanding the market and the dynamic spreading of shocks, and, hence, may be used for policy and regulatory analysis.

We find that there are strong direct links between the big four Australian banks, which are connected to the real economy, real estate, and financial groups. The Australian market is also seen to be strongly connected to the Asian market. The reconstructed network can be partly explained by the network of technological input requirements for the considered sectors.

The rest of this paper is organized as follows. Section 2 defines the network of partial correlations. Section 3 discusses key network measures and interprets them for the network of partial correlations. In particular, we highlight a connection between the eigenvalue centrality and the principal component analysis. Section 4 details the estimation procedure. Section 5 applies the network methods to uncover a perceived network of the Australian banks including connections to local financial and real sectors, and global 
markets, and demonstrate relevant policy examples. We also compare the reconstructed perceived networks with the actual networks of direct input requirements based on the input-output tables. Section 6 concludes the paper.

\section{Networks of partial correlations}

Formally, a graph $\mathcal{G}=(\mathcal{V}, \mathcal{E})$ is a structure consisting of a set of nodes, $\mathcal{V}$, and a set of edges, $\mathcal{E}$. Every two nodes may or may not be connected by an edge, edges may be directed or undirected, and weighted or unweighted. Our focus here is on undirected graphs and, hence, elements of an edge set $\mathcal{E}$ are unordered pairs $(i, j)$ of distinct nodes $i, j \in \mathcal{V}$. We are working with weighted graphs where each edge has a non-zero weight $w_{i j}$ assigned to it.

Following the literature on Gaussian graphical models (e.g., Whittaker, 2009), we define the network of partial correlations. Let $X$ denote an $n$-dimensional multivariate random variable and the nodes of the graph $\mathcal{G}$ correspond to each element of $X$, i.e., $\mathcal{V}=\left\{X_{1}, X_{2}, \ldots, X_{n}\right\}$. Let $X_{i \mid \mathcal{V} \backslash\left\{X_{i}, X_{j}\right\}}$ denote the best linear approximation of variable $X_{i}$ based on all the variables except for $X_{i}$ and $X_{j}$ for any pair $i, j$.

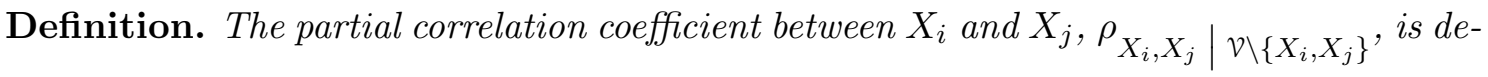
fined as the ordinary correlation coefficient between $X_{i}-X_{i \mid \mathcal{V} \backslash\left\{X_{i}, X_{j}\right\}}^{*}$ and $X_{j}-X_{j \mid \mathcal{V} \backslash\left\{X_{i}, X_{j}\right\}}^{*}$.

In other words, the partial correlation between $X_{i}$ and $X_{j}$ is equal to the correlation between the residuals of the two linear regressions: (1) $X_{i}$ on a constant and a set of control variables, which includes all variables in $X$ except for $X_{i}$ and $X_{j}$, and (2) $X_{j}$ on a constant and the same set of control variables as in the first regression. Hence, the partial correlation measures linear dependence between any two elements of $X, X_{i}$ and $X_{j}$, for $i \neq j$, after controlling for linear dependence with all other remaining elements in $\mathcal{V} \backslash\left\{X_{i}, X_{j}\right\}$. For the sake of brevity, we will use a shorter notation $\rho_{i j \mid}$. for the partial correlation between $X_{i}$ and $X_{j}$.

The edges of the network of partial correlations correspond to the pairs of random variables with non-zero partial correlations, $\mathcal{E}=\left\{(i, j) \in \mathcal{V} \times \mathcal{V} \mid \rho_{i, j \mid} \neq 0\right\}$, and the edge weights to the corresponding partial correlations, $w_{i j}=\rho_{i j \mid}$. Intuitively, the network of partial correlations visualizes linear dependence between any two random variables conditional on all other variables. When the random variable $X$ is multivariate normal, zero 
partial correlation implies conditional independence of the corresponding elements. This statement holds for a more general case of an arbitrary continuous marginal distribution for each element of $X$ when the dependence between the elements is characterized by the Gaussian copula (see, e.g., Diks et al., 2010, 2014).

For better intuition behind the concept of partial correlations, it is useful to show connections with linear regression. Project each $X_{i}, 1 \leq i \leq n$ on the space spanned by the rest of the variables in $X$ as

$$
X_{i}-m_{i}=\sum_{j \neq i} \beta_{i j}\left(X_{j}-m_{j}\right)+\varepsilon_{i}
$$

where $m_{i}$ is the unconditional mean of $X_{i}$ and $\varepsilon_{i}$ is a zero-mean residual. Denote the variance-covariance matrix of vector of the residuals, $\varepsilon$, as $\boldsymbol{\Sigma}$. This matrix is not necessarily diagonal as $\varepsilon$ 's may be correlated. The diagonal elements of $\boldsymbol{\Sigma}$ are the conditional variances of the elements of $X, \operatorname{Var}\left(\varepsilon_{i}\right)=\operatorname{Var}\left(X_{i} \mid \mathcal{V} \backslash\left\{X_{i}\right\}\right)$. The orthogonality condition, $E\left(\varepsilon_{i} X_{j}\right)=0, \forall j \neq i, 1 \leq i, j \leq n$, implies that regression coefficients are given by $\beta_{i j}=\rho_{i j \mid} \cdot \sqrt{\operatorname{Var}\left(\varepsilon_{i}\right) / \operatorname{Var}\left(\varepsilon_{j}\right)}$ (see Appendix A.1). It follows that $\rho_{i j \mid}=\operatorname{sign}\left(\beta_{i j}\right) \sqrt{\beta_{i j} \beta_{j i}}$.

Partial correlations are also related to the (unconditional) variance-covariance matrix of $X, \boldsymbol{\Omega}=\operatorname{Cov}(X)$. Define a concentration or precision matrix as the inverse of a nonsingular variance-covariance matrix, $\mathbf{K} \equiv \boldsymbol{\Omega}^{-1}$. The partial correlations can be expressed as

$$
\rho_{i j \mid}=\frac{-k_{i j}}{\sqrt{k_{i i} k_{j j}}},
$$

where $k_{i j}$ is the $(i, j)$ entry of $\mathbf{K}$ (see Appendix A.2). Furthermore, each diagonal element of $\mathbf{K}$ is the reciprocal of a conditional variance, i.e., $k_{i i}=1 / \operatorname{Var}\left(\varepsilon_{i}\right)$, where $\varepsilon_{i}$ is defined in Eq. (1).

Finally, the partial correlation can also be computed using the inverse of $\mathbf{R}$, the regular correlation matrix of $X$, by replacing the $k_{i j}, k_{i i}$, and $k_{j j}$ entries in Eq. (2) with the corresponding entries of $\mathbf{R}^{-1}$ (see Appendix A.3). The $i$ th diagonal element of $\mathbf{R}^{-1}$ is the ratio of unconditional variance of $X_{i}, \operatorname{Var}\left(X_{i}\right)$, to conditional variance of $X_{i}, \operatorname{Var}\left(\varepsilon_{i}\right)$. The proportion of variation in $X_{i}$ as explained by all the other elements can be defined, similarly to the regression's coefficient of determination, as

$$
R_{i}^{2}=1-\frac{\operatorname{Var}\left(\varepsilon_{i}\right)}{\operatorname{Var}\left(X_{i}\right)}
$$


and deduced from the diagonal elements of $\mathbf{R}^{-1}$. This measure can be thought of as an endogenous network-induced variation of $X_{i}$.

A graph with $n$ nodes can be represented by the adjacency matrix of size $n \times n$. When the graph is undirected and weighted, as in our case, the adjacency matrix is a symmetric matrix whose $(i, j)$-entry is non-zero only if there is an edge connecting nodes $i$ and $j$, and the entry is given by the weight. As we will see in the next Section, adjacency matrix is a basis for other network-based measures.

Let $\mathbf{P}$ denote the adjacency matrix of the graph of partial correlations. The elements of this matrix are $\mathbf{P}_{i, j}=\rho_{i j \mid}$. for $i \neq j$ and zeros on the diagonal. From (2) we have

$$
\mathbf{P}=\mathbf{I}-\mathbf{D}_{\mathbf{K}}^{-1 / 2} \mathbf{K D}_{\mathbf{K}}^{-1 / 2}
$$

where $\mathbf{I}$ is the identity matrix of size $n, \mathbf{K}$ is the concentration matrix, and $\mathbf{D}_{\mathbf{K}}$ is the diagonal matrix composed of the diagonal elements of $\mathbf{K}$. The diagonal elements of $\mathbf{K}$ are the inverse of the conditional variances of the elements of $X$. Therefore,

$$
\mathbf{D}_{\mathbf{K}}=\operatorname{diag}\left\{k_{11}, \ldots, k_{n n}\right\}=\operatorname{diag}\left\{\frac{1}{\operatorname{Var}\left(\varepsilon_{1}\right)}, \ldots, \frac{1}{\operatorname{Var}\left(\varepsilon_{n}\right)}\right\}=\mathbf{D}_{\mathbf{\Sigma}}^{-1}
$$

where $\mathbf{D}_{\boldsymbol{\Sigma}}$ is the diagonal matrix composed of the diagonal elements of $\boldsymbol{\Sigma}$.

Next, we show how the adjacency matrix $\mathbf{P}$ relates to the system of linear equations (1). Introduce the matrix $\mathbf{B}$ with zeros on the diagonal and $\beta_{i j}$ in the $(i, j)$ offdiagonal entry. Exploring the relationship between $\rho_{i j \mid}$. and $\beta_{i j}$ defined above, we find that $\mathbf{B}=\mathbf{D}_{\boldsymbol{\Sigma}}^{1 / 2} \mathbf{P} \mathbf{D}_{\boldsymbol{\Sigma}}^{-1 / 2}$. Then Eq. (1) can be rewritten in a matrix form as follows

$$
X-m=\mathbf{B}(X-m)+\varepsilon=\mathbf{D}_{\Sigma}^{1 / 2} \mathbf{P D}_{\boldsymbol{\Sigma}}^{-1 / 2}(X-m)+\varepsilon,
$$

where $m$ and $\varepsilon$ are the vectors of the means of $X$ and the residuals, respectively. While we should be careful about causal interpretation of this equation, it is useful to think about $\varepsilon$ 's as external shocks influencing the system. Because $X_{i}$ 's may have different conditional variances, to compare the effects of the shocks we rescale the residuals in such a way that all of them would have unit conditional variance, $e=\mathbf{D}_{\Sigma}^{-1 / 2} \varepsilon$. It is important to emphasize that $e$ 's are not independent (neither are the entries of $\varepsilon$ ).

We multiply both sides of the previous equation by $\mathbf{D}_{\Sigma}^{-1 / 2}$ from the left and introduce 
a rescaled variable $x=\mathbf{D}_{\Sigma}^{-1 / 2}(X-m)$. The equation becomes

$$
x=\mathbf{P} x+e .
$$

Note that the rescaling for $X$ is in terms of the conditional variance as opposed to a more usual rescaling by the unconditional variance. Intuitively, by using the conditional variance we remove the effect of the variables endogenous to the network.

In the next section, we will use the eigenvalues and eigenvectors of matrix $\mathbf{P}$. Note that $\mathbf{P}$ is the real symmetric matrix. Therefore, it has a set of $n$ linearly independent eigenvectors corresponding to the real eigenvalues. ${ }^{2}$ We order the eigenvalues by their absolute values as $\left|\lambda_{1}\right| \geq\left|\lambda_{2}\right| \geq \cdots \geq\left|\lambda_{n}\right|$. The spectral radius of the matrix $\mathbf{P}$ is defined as $\rho(\mathbf{P})=\left|\lambda_{1}\right|$, the largest absolute eigenvalue. It is important to make the following assumption for further exposition.

Assumption 1. The spectral radius of the adjacency matrix of the graph of partial correlations, $\mathbf{P}$, is less than 1 .

This Assumption is satisfied when all entries of $\mathbf{P}$ are non-negative. Moreover, it holds more generally, when all the row sums of the absolute values of the elements of $\mathbf{P}$ are less than 1. In other words, Assumption 1 holds when partial correlation matrices exhibit moderate dependence. In particular, this turned out to be true in all our applications. Finally, we note that Assumption 1 is equivalent to the condition where the smallest eigenvalue of $\mathbf{P}$ is larger than -1 . Formal proofs of these statements can be found in Appendix A.4.

We now demonstrate the usefulness of the network of partial correlations for the systemic risk analysis, and its connection with the network of correlations.

\subsection{Interpretation of Partial Correlation Network}

In Section 5 we reconstruct the perceived financial networks of the Australian banks, other sectors of the economy, and international markets using publicly available information on the returns of the corresponding bank shares and indexes. The returns reflect market perception about the percentage change in the present value of a company, sector, or

\footnotetext{
${ }^{2}$ Note that the eigenvalues have different signs. It immediately follows from the fact that the sum of all eigenvalues of $\mathbf{P}$ is equal to the trace of $\mathbf{P}$, which is zero. As a consequence, matrix $\mathbf{P}$ is indefinite. This is an important difference from the correlation matrices, which are always positive definite.
} 
marker overall. By looking at the correlations of the returns for some entities, we may uncover how the market perceives joint changes in the value of these entities, including any intermediate effects. The use of correlations in this sense has been widely used in finance for optimal portfolio selection (Markowitz, 1952). However, if one wants to understand the structure of the market and use it for, say, financial stability analysis or optimal policy design, it is important to turn to partial correlation analysis. Partial correlations can single out the direct co-movements in the relative change of values between the pairs of entities, while controlling for all other entities. By reconstructing the network of partial correlations, we may observe how a unit variance shock may spread through the network. From the perspective of the regulator, this allows identifying the most important relations and focusing policy on these relations or mitigating possible consequences of any large shocks to these entities.

It is important to emphasize at this point that with partial correlations, it is not possible to establish the direction of causation. The concept of Granger causality (Granger, 1969) can be used to establish directional relationships within the VAR framework (see, e.g., Billio et al., 2012). However, the presence of Granger causality would imply predictability in returns which the market should incorporate. Therefore, unless we turn to high frequencies, it is hardly possible to detect substantial and stable linear Granger causality in financial returns. Lower frequency cross-sectional dependencies include Granger causalities accumulated at higher frequencies (see, e.g., Barigozzi and Brownlees, 2013).

With this caution about causality in mind, we propose an observational interpretation of system (5). The expected steady state value of $x$ is 0 . Suppose that we observe a deviation in $x$. We will now decompose the total observed deviation into a direct effect given by unit-variance shock $e$ and an indirect (endogenous to the network) effect. An initial shock $e_{i}$ which directly hits node $X_{i}$ will also affect the immediate neighbors of $X_{i}$. For the network as a whole, the expected effect of shock $e$ on the immediate neighbors is measured by $\mathbf{P} e$. The $i$ th element of this vector, given by $\sum_{j=1}^{n} \rho_{i j \mid} \cdot e_{j}$, accumulates the effect of the initial shocks to all other nodes reaching node $X_{i}$ in one step. We call this a first-order effect. The expected effect on the neighbors of the neighbors can be computed as $\mathbf{P}(\mathbf{P} e)=\mathbf{P}^{2} e$, which we call a second-order effect. Generally, we define a $k$ th-order effect as $\mathbf{P}^{k} e$. It gives the effect of the initial shock after traveling $k$ steps along the edges 
of the network of partial correlations, summed over all possible walks. ${ }^{3}$ The total effect of the shock on $x$ will now be

$$
e+\mathbf{P} e+\mathbf{P}^{2} e+\cdots=\sum_{k=0}^{\infty} \mathbf{P}^{k} e
$$

when this sum converges. Assumption 1 is necessary and sufficient for convergence, ${ }^{4}$ in which case $\sum_{k} \mathbf{P}^{k}=(\mathbf{I}-\mathbf{P})^{-1}$. Using Eq. (4), one can see that $(\mathbf{I}-\mathbf{P})^{-1}$, denoted by $\mathbf{T}$, is equal to

$$
\mathbf{T}=\mathbf{D}_{\Sigma}^{-1 / 2} \Omega \mathbf{D}_{\Sigma}^{-1 / 2}
$$

so that the total effect of the shock is given by $\mathbf{T} e$. The matrix $\mathbf{T}$ is a variance-covariance matrix of $x$, the rescaled $X$. Eq. (7) shows that it is also the rescaled variance-covariance matrix $\boldsymbol{\Omega}$. It looks similar to an ordinary correlation matrix, but, instead of using unconditional variances of $X$ for rescaling, conditional variances of $X$ are used.

We have established that $\mathbf{T}$ transforms the initial shock into its total effect on $X$. Instead, the matrix of partial correlations $\mathbf{P}$ defines how shocks spill over on the immediate neighbors.

\section{Network-based Measures of Centrality}

The previous interpretation shows that the network of partial correlations can be used to separate the direct and higher order spillover effects of shocks. Understanding and measuring spillovers by means of the partial correlation matrix, as opposed to limiting attention to the variance-covariance matrix relevant for the total effect of the shock, is important for a policy aiming to reduce systemic risk. Some edges of the partial correlation network (intuitively, those with high weights) and, as a consequence, some nodes (namely, those with many edges with high weights), may play a higher role in these spillover effects.

\footnotetext{
${ }^{3} \mathrm{~A}$ walk in a graph is a sequence of (possibly repeated) nodes and edges that begins and ends with nodes. A walk of length $k$ has $k$ edges. Thus, the $k$ th order impact of the initial shock $e_{j}$ on $X_{i}$ is the sum over all possible walks of length $k$ starting in node $j$ and finishing in node $i$ of the products of all partial correlations along the walk times $e_{j}$. The $i$ th element of the vector $\mathbf{P}^{k} e$ is then a sum of these $k$ th order impacts over all nodes (including $i$ ).

${ }^{4}$ Note that this Assumption is equivalent to $\lim _{k \rightarrow \infty} \mathbf{P}^{k}=\mathbf{0}$. The $(i, j)$ element of matrix $\mathbf{P}^{k}$ sums, over all possible walks of length $k$ from $i$ to $j$, the products of partial correlations of the edges of this graph along the walks. All these products converge to 1 , but the number of walks explodes. Assumption 1 guarantees that the former effect dominates the latter. It shows that our interpretation works whenever the values of partial correlations are moderate, so that traveling over the network will sufficiently dampen the shocks.
} 
Network theory uses various measures of centrality to measure the relative importance of nodes in the graph.

There exist several different centrality measures that attempt to evaluate the nodes' positions on the graph. One of the simplest centrality measures for a graph is degree centrality. For a weighted graph, degree centrality is defined for each node by adding all weights of the edges connected to the given node. In our case, for the adjacency matrix $\mathbf{P}=\left(\mathbf{P}_{i, j}\right)_{i, j=1}^{n}$ in the partial correlation network, the node's degree is computed as

$$
c_{i}^{\mathrm{D}}=\mathbf{P} \cdot \mathbf{1}=\sum_{j=1}^{n} \mathbf{P}_{i, j}=\sum_{j=1}^{n} \rho_{i j \mid},
$$

where $\mathbf{1}$ is an $n \times 1$ vector of ones, and $c^{\mathrm{D}}$ is a vector of degree centralities. Intuitively, the nodes with high degree centrality (in absolute value) are important for the transmission of the shock to/from ${ }^{5}$ the immediate neighbors because they have many edges and/or edges with high weights. ${ }^{6}$

To analyze the higher order impacts of the initial shock, we need to use self-referential centrality measures, i.e., when high centrality is assigned to the nodes connected to other central nodes. One such measure is eigenvector centrality, for which every element $c_{i}^{\mathrm{E}}$ is proportional to $\sum_{j=1}^{n} \rho_{i j \mid} \cdot c_{j}^{\mathrm{E}}$, or, formally, vector $c^{\mathrm{E}}$ is an eigenvector of matrix $\mathbf{P}$. Which of the $n$ eigenvectors is taken as the eigenvector centrality ultimately depends on the nature of the network and resulting interpretation. ${ }^{7}$ We will argue now that for the network of partial correlations, when the largest absolute eigenvalue of $\mathbf{P}$ is unique, the corresponding eigenvector can be taken as the eigenvector centrality.

Let us apply operator $\mathbf{P}$ iteratively to an arbitrary non-zero initial vector $e_{0} \in \mathbb{R}^{n}$. The symmetric real matrix $\mathbf{P}$ has the orthonormal basis $\left\{u_{1}, \ldots, u_{n}\right\}$ of the eigenvectors corresponding to the eigenvalues $\left|\lambda_{1}\right|>\left|\lambda_{2}\right| \geq \cdots \geq\left|\lambda_{n}\right|$. Writing $e_{0}$ in this basis with

\footnotetext{
${ }^{5}$ The direction of the shock is not explicitly observed because the network of partial correlations is undirected.

${ }^{6}$ Note that some entries of $\mathbf{P}$ may have a negative value, indicating that the sign of the shock will be reversed for the corresponding nodes. If the node has some edges with positive weights and some edges with negative weights, the effects will cancel each other out, resulting in a small overall impact of the node. In this case, it is useful also to consider $\overline{\mathbf{P}}$, the element-wise absolute value of matrix $\mathbf{P}$. For this matrix, the degree centrality will indicate the importance of the node in the transmission of both positive and negative shocks. In our application we find that the number of edges with negative partial correlations is small and the numerical values of the negative partial correlations are negligible. Therefore, we do not focus on centrality measures for $\overline{\mathbf{P}}$.

${ }^{7}$ The network literature typically works with non-negative matrices (i.e., having non-negative entries). Non-negative matrices have a unique non-negative eigenvector and this vector is taken as the eigenvector centrality. As $\mathbf{P}$ is not necessarily non-negative, such a criterion is not sufficient for us.
} 
coordinates $\left\{b_{1}, \ldots, b_{n}\right\}$ and then applying $\mathbf{P}^{k}$, we obtain

$$
\mathbf{P}^{k} e_{0}=\mathbf{P}^{k} \sum_{\ell=1}^{n} b_{\ell} u_{\ell}=\sum_{\ell=1}^{n} b_{\ell} \mathbf{P}^{k} u_{\ell}=\sum_{\ell=1}^{n} b_{\ell} \lambda_{\ell}^{k} u_{\ell}=b_{1} \lambda_{1}^{k} u_{1}+\sum_{\ell=2}^{n} b_{\ell}\left(\frac{\lambda_{\ell}}{\lambda_{1}}\right)^{k} u_{\ell} .
$$

Since $\left|\lambda_{\ell} / \lambda_{1}\right|<1$ for all $\ell \geq 2$, the last sum converges to 0 as $k \rightarrow \infty$. Therefore, the eigenvector $u_{1}$ gives the asymptotic direction for $\mathbf{P}^{k} e_{0}$ when $k \rightarrow \infty$ for any $e_{0}$ which is not orthogonal to $u_{1}$. If we interpret $e_{0}$ as a vector of shocks affecting the values of nodes in the graph, then the eigenvector $u_{1}$ corresponding to the largest absolute eigenvalue $\lambda_{1}$ characterizes the asymptotic impact ( $k$-th order when $k \rightarrow \infty$ ) of an initial shock on the nodes. This property allows us to consider vector $u_{1}$ as the eigenvector centrality measure, $c^{\mathrm{E}}$. As any eigenvector is defined up to a multiplicative constant, the nominal values of the elements of $c^{\mathrm{E}}$ are not important by themselves. The relative values of elements of this vector reflect the relative asymptotic impact of the initial shock on the corresponding nodes.

In case of multiple largest absolute eigenvalues, the last sum in Eq. (8) will not converge to zero. In such situations, the shock will asymptotically belong to the space spanned by all the eigenvectors corresponding to the largest absolute eigenvalues. Moreover, even when there is a unique largest absolute eigenvalue, the convergence of the last sum in (8) may be slow when the second largest absolute eigenvalue is close to the first (see Section 11.1.1 in Newman, 2010). It motivates the introduction of the p-eigenvector centrality space of dimension $p<n$ as the space spanned by the $p$ eigenvectors corresponding to the $p$ largest absolute eigenvalues. When the largest $p$ absolute eigenvalues are large relative to the remaining eigenvalues, but close to each other, this space will provide a better asymptotic approximation for convergence of the initial shock.

This approach resembles the principle component analysis (PCA), and the following proposition allow us to establish the link between $p$-centrality space for the matrix $\mathbf{P}$ and the PCA applied to the matrix $\mathbf{T}$ defined in (7).

Proposition 1. Let $\lambda$ be an eigenvalue of the adjacency matrix $\mathbf{P}$ of the graph of partial correlations with corresponding eigenvector $u$. Then $1 /(1-\lambda)$ is the eigenvalue of matrix $\mathbf{T}$, the variance-covariance matrix of $x$, defined in (7), with the same corresponding eigenvector $u$. 
Proof. The statement follows from the following chain of equivalence relations:

$$
\begin{aligned}
\mathbf{P} u=\lambda u & \Leftrightarrow u-\mathbf{D}_{\mathbf{K}}^{-1 / 2} \mathbf{K D}_{\mathbf{K}}^{-1 / 2} u=\lambda u \quad \Leftrightarrow \\
& \Leftrightarrow \quad \mathbf{D}_{\mathbf{K}}^{-1 / 2} \mathbf{K D}_{\mathbf{K}}^{-1 / 2} u=(1-\lambda) u \quad \Leftrightarrow \quad \mathbf{D}_{\mathbf{\Sigma}}^{1 / 2} \mathbf{\Omega}^{-1} \mathbf{D}_{\mathbf{\Sigma}}^{1 / 2} u=(1-\lambda) u \quad \Leftrightarrow \\
& \Leftrightarrow \mathbf{T}^{-1} u=(1-\lambda) u \quad \Leftrightarrow \quad \frac{1}{1-\lambda} u=\mathbf{T} u .
\end{aligned}
$$

The last Proposition asserts that the matrix $\mathbf{P}$ has the same eigenvectors as the rescaled variance-covariance matrix $\mathbf{T}$, and even if their eigenvalues differ, their ordering does not. If $p$ largest absolute eigenvalues are positive, the space to which the first $p$ principle components of $\mathbf{T}$ belong will coincide with the $p$-eigenvector centrality space of $\mathbf{P}$.

Finally, we discuss another centrality measure which is often found relevant in the economic literature: the so-called Bonacich centrality. ${ }^{8}$ As the eigenvector centrality, it is defined in a self-referential manner but also reflects the degree centrality. Specifically, $c_{i}^{\mathrm{B}}=\alpha \sum_{j=1}^{n} \rho_{i j \mid} \cdot c_{j}^{\mathrm{B}}+c_{i}^{\mathrm{D}}$. The weight $\alpha$ measures the importance of the centrality of the neighbors with respect to one's own degree centrality. In matrix form, this definition reads

$$
c^{\mathrm{B}}=\alpha \mathbf{P} c^{\mathrm{B}}+\mathbf{P} \cdot \mathbf{1} \quad \Leftrightarrow \quad c^{\mathrm{B}}=(\mathbf{I}-\alpha \mathbf{P})^{-1} \mathbf{P} \cdot \mathbf{1},
$$

where, as before, $\mathbf{1}$ is an $n \times 1$ vector of ones and $\mathbf{I}$ is the identity matrix of size $n$. When $\alpha=0$ the Bonacich centrality is simply the degree centrality, i.e, the first-order effect of the unit shock $e=\mathbf{1}$. For any $\alpha \leq 1$, Assumption 1 allows us to rewrite the Bonacich centrality as $c^{\mathrm{B}}=\mathbf{P} \cdot \mathbf{1}+\alpha \mathbf{P}^{2} \cdot \mathbf{1}+\alpha^{2} \mathbf{P}^{3} \cdot \mathbf{1}+\ldots$ However, intermediate values of $\alpha \in(0,1)$ are not particularly important in our application. ${ }^{9}$ When $\alpha=1$, the Bonacich centrality is equal to the cumulative of the first-, second-, and all higher order effects of the unit

\footnotetext{
${ }^{8}$ Acemoglu et al. (2012) derive the expression for Bonacich centrality in the context of intersectoral (input-output) network. Ballester et al. (2006) relate the Bonacich centrality to the actions in the Nash equilibrium of noncooperative game. The definition given here is a special case of the measure proposed in Bonacich (1987). There, for the network with adjacency matrix $\mathbf{A}$, the centrality is defined as $c^{\mathrm{B}}(\alpha, \beta)=\beta(\mathbf{I}-\alpha \mathbf{A})^{-1} \mathbf{A} \cdot \mathbf{1}$. The constant $\beta$ scales the centralities of all the modes and here we assume $\beta=1$.

${ }^{9}$ The Bonacich centrality measure was introduced for social networks where the edges are directly observed and the cumulative effect of the interactions is of interest. Dampening is a reasonable assumption for this setup. In our case, the network of partial correlations is obtained from the variance-covariance matrix, which already gives us the cumulative effect. The dampening is already accounted for in matrix $\mathbf{P}$.
} 
shock $e=\mathbf{1}$, as

$$
c^{\mathrm{B}}=(\mathbf{I}-\mathbf{P})^{-1} \mathbf{P} \cdot \mathbf{1}=\mathbf{P} \cdot \mathbf{1}+\mathbf{P}^{2} \cdot \mathbf{1}+\mathbf{P}^{3} \cdot \mathbf{1}+\ldots=\mathbf{T} \cdot \mathbf{1}-\mathbf{1},
$$

where the latter equality stems from Eq. (6). In this way we establish a direct link between Bonacich centrality and the initial variance-covariance matrix, $\boldsymbol{\Omega}$, via its rescaling $\mathbf{T}$.

To summarize, in this section we introduced three measures of centrality. Each measure highlights a particular aspect of shock propagation. Degree centrality identifies the nodes relevant for transmission of shocks to the immediate neighbors and for the first-order effects of unit shock. Eigenvector centrality (and, more generally, $p$-eigenvector centrality space) is relevant for describing the asymptotic distribution of shocks. Bonacich centrality identifies the nodes hit by a cumulated shock.

\section{Estimation procedure for time series}

In Section 2 we have defined the network of partial correlations for an $n$-variate random variable. Now we show how to construct partial correlations from an $n$-variate time series process. This is done by modeling serial dependence possibly present in time series data so that after filtering it out we obtain a serially independent $n$-variate random variable.

In our application we will use the financial return series. A well-known stylized fact of the financial time series is volatility clustering. GARCH-type specifications (see, e.g., Bollerslev et al., 1992) are typically used to model conditional volatility. ${ }^{10}$ There are many multivariate GARCH specifications available (see a survey of Bauwens et al., 2006), but the most flexible multivariate models require estimating an infeasibly large number of parameters. We select a parsimonious specification, the constant conditional correlations (CCC) model by Bollerslev (1990). ${ }^{11}$ In the CCC model, the conditional means and conditional variances are modeled separately for each dimension using univariate models, and then correlations are estimated from the filtered series. Denote an $n$-variate time

\footnotetext{
${ }^{10}$ There are other models for conditional volatility, e.g., stochastic volatility models (Kim et al., 1998) or recently introduced GAS models (Creal et al., 2013). Even within the class of ARCH models one may choose a number of different volatility specifications and make various distributional assumptions (see, e.g., Bao et al., 2007). These choices are important in risk management and conditional volatility forecasting (Diks et al., 2011), but not in our context.

${ }^{11}$ Another popular and more flexible specification is the dynamical conditional correlations (DCC) model of Engle (2002). We have implemented this specification as well, but the daily changes in partial correlations were very small relative to the overall average level. The estimates of the partial correlations we obtained with the DCC model were similar to those obtained with the CCC model.
} 
series as $Y_{t}=\left(Y_{1, t}, \ldots, Y_{n, t}\right)^{\prime}$. Formally the model is specified as

$$
Y_{t}=\mu_{t}+\sqrt{H_{t}} \epsilon_{t}
$$

where $\mu_{t}=\left(\mu_{1, t}, \ldots, \mu_{n, t}\right)^{\prime}$ is a specification of the conditional mean, $H_{t}=\operatorname{diag}\left(h_{1, t}, \ldots, h_{n, t}\right)$ is the conditional variance, and $\epsilon_{t}=\left(\epsilon_{1, t}, \ldots, \epsilon_{n, t}\right)^{\prime}$ are the standardized innovations, assumed to be serially independent, and identically normally distributed with each element having zero mean and unit variance, but cross-sectionally dependent, with a constant correlation matrix R. For simplicity, we assume $\operatorname{ARMA}(1,1)$ and $\operatorname{GARCH}(1,1)$ specifications for the conditional means and variances, respectively, ${ }^{12}$ so that

$$
\begin{aligned}
& \mu_{i, t}=c_{i}+\phi_{i} Y_{i, t-1}+\theta_{i}\left(Y_{i, t-1}-\mu_{i, t-1}\right), \\
& h_{i, t}=\omega_{i}+\alpha_{i}\left(Y_{i, t}-\mu_{i, t}\right)^{2}+\gamma_{i} h_{i, t-1} .
\end{aligned}
$$

The correlation matrix can now be estimated from the estimates of the standardized innovations as $\widehat{\mathbf{R}}=\frac{1}{T} \sum_{t=1}^{T} \widehat{\epsilon}_{t} \widehat{\epsilon}_{t}^{\prime}$, where $\widehat{\epsilon}_{i, t}=\left(Y_{i, t}-\widehat{\mu}_{i, t}\right) \widehat{h}_{i, t}^{-1 / 2}$ and $T$ is a sample size.

From the correlation matrix $\widehat{\mathbf{R}}$, the matrix of partial correlations, $\widehat{\mathbf{P}}$, is obtained using Eq. (2). If $T$ is small and the number of considered variables $n$ is large, this procedure may become unstable as $\widehat{\mathbf{R}}$ may be ill-conditioned. ${ }^{13}$ In this case shrinkage or penalized maximum likelihood estimators are handy. Given that our sample size is sufficiently large relative to the matrix dimension, we do not experience this problem. However, as a robustness check we implement a shrinkage-based glasso estimator by Peng et al. (2009) and report these results in Appendix E.

\section{Empirical application}

The network setup described above may be applied in many different contexts. In this section we use this setup to uncover the perceived network of the Australian banks and in-

\footnotetext{
${ }^{12}$ Formal model selection criteria, e.g., AIC, can be used to choose an optimal order for ARMA and GARCH processes. Typically, parsimonious low-order models are selected. Normality of the standardized innovations may also be relaxed (Lee and Long, 2009). Note that in our specific application, conditional mean and volatility filtering are not important in the sense that the partial correlations obtained from the raw returns are similar to those obtained after $\operatorname{ARMA}(1,1)-\mathrm{GARCH}(1,1)$ filtering in the CCC model.

${ }^{13}$ The conditioning number for a symmetric positive definite matrix is defined as the ratio of its maximum and minimum eigenvalues, $k=\lambda_{\max } / \lambda_{\min }$. In case of the large conditioning number, the matrix is said to be ill-conditioned and the computation of its inverse is subject to large numerical errors (Belsey et al., 1980).
} 
dustries. We use the term "perceived" to emphasize that our analysis is based solemnly on the returns of publicly traded banks and sectors, and can reveal the network of connections implied only by the market-driven return co-movements.

Our sample spans the period from 6 November 2000 to 22 August 2014 and was obtained from Datastream with 3,600 daily observations in total. We have identified 8 publicly traded banks in the most recent period: the "big four" (ANZ, CBA, NAB, Westpac), two regional banks (Bank of Queensland, and Bendigo and Adelaide Bank) and two large financial groups providing banking services among others (Suncorp and Macquarie group). We also look at the index returns of two major financial sub-sectors other than banking: Insurance and Real Estate. Additionally, we include 9 major sectors of the Australian economy, i.e., Basic materials, Industrials and others. The sectoral classification is based on the Industry Classification Benchmark and is provided by Datastream (see Appendix B for details). Finally, we include the Datastream Asian market index, which includes major Asian companies, given the region's close links to the Australian economy.

Initially the CCC model was estimated using the full 2000-2014 sample of 3,600 days. Later we divided the sample into two subsamples of roughly equal lengths: "pre-2008" covering 2000-2007 with 1, 868 observations and "post-2008" covering 2008-2014 with 1, 732 observations. These may be thought of as pre-crisis and post-crisis subsamples, respectively.

Appendix C reports the matrices of correlations, $\widehat{\mathbf{R}}$, and partial correlations, $\widehat{\mathbf{P}}$, for the full sample. There are multiple instances when two entities with relatively high correlation exhibit low partial correlation. For example, the correlation between NAB and Westpac is 0.65 , whereas the partial correlation is only 0.1 , indicating that NAB and Westpac have relatively strong indirect connections.

Figure 1 shows the reconstructed network of partial correlations. The thickness of the edges corresponds to the strength of the partial correlations. For improved visibility, we omit edges corresponding to partial correlations smaller than 0.075 by absolute value. ${ }^{14}$ The banks and other financial institutions are collected in the lower part of the graph. We notice strong partial correlations between the big four banks and their links with other banks, and with the financial and real economy sectors. Interestingly, the Macquarie group acts as a link between the banking sector and the real economy and Asia. This is likely

\footnotetext{
${ }^{14}$ All negative entries were far below this threshold, so that all shown edges have positive weights.
} 


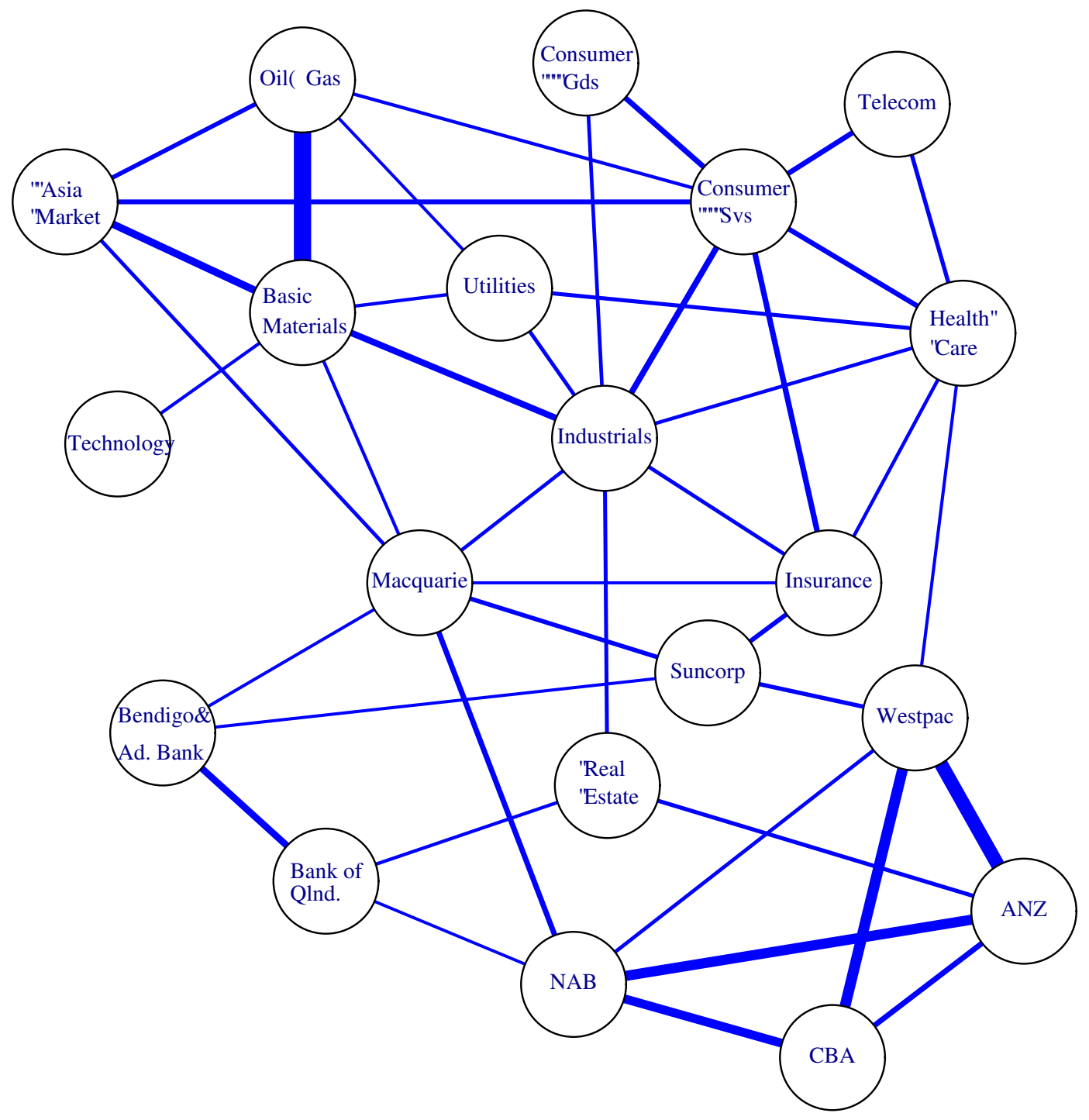

Figure 1: Network of partial correlations of the Australian banks and other sectors. Full sample of 3,600 days was used for estimation. 
due to the fact that Macquarie is the largest Australian investment bank, which provides M\&A advisory services and has a large presence in the Asian region. ANZ and Bank of Queensland have strong direct links with the Real Estate subsector. Bank of Queensland consistently reported large exposures concentrated in Real Estate. ${ }^{15}$ The regional banks, Bank of Queensland and the Bendigo and Adelaide Bank, have a strong direct link. The former is directly linked with NAB, while the latter is directly linked with the Macquarie group and Suncorp. ${ }^{16}$ Suncorp operates both insurance and banking businesses, which explains its links with the Insurance sector and other banks. The Industrials and Consumer Services sectors seem to be in the center of the Australian economy. The Asian market influences the Australian economy primarily through Basic materials, Oil \& Gas, and Consumer Services. ${ }^{17}$

The two upper panels in Figure 2 show the networks of partial correlations for pre-2008 and post-2008 subsamples. We notice that some connections have changed substantially pre- and post-2008. Note that the estimates for subsamples are subject to a higher estimation noise due to a smaller sample size compared to the full sample. Interestingly, the interbank connections and connections between the banks and other sectors have increased post-2008. At the same time we note a decrease in the central role of Basic Materials and an increase in the central role of Industrials post-2008.

We suggested reasons for several strong links between the financial entities in the network of partial correlations. The observed connections between sectors may also arise from interdependencies induced by technological processes. For illustration purposes we provide an example of an economic network capturing these interdependencies, namely the network based on the flows of goods and services between the sectors. The two lower panels of Figure 2 show the IO networks, which reflect the direct technological requirements and are derived from the Input-Output tables for the tax years 2006-2007 and 2009-2010, respectively. ${ }^{18}$ These periods roughly correspond to the pre-2008 and

\footnotetext{
${ }^{15}$ BoQ large exposures to Property and Construction sectors were around 80\% in 2011-2008 as reported in their presentations to investors. Source: http://www.boq.com.au/shareholder_investor.htm

${ }^{16}$ Possible explanations for some of these links may stem from a proposed, but not realized, merger between the Bendigo and Adelaide Bank and BoQ in 2007 (source: http://www.boq.com.au/uploadedFiles/ BOQ_merger_proposal_Investor_Presentation.pdf) and the fact that the Bendigo and Adelaide Bank acquired Macquarie Group's margin lending portfolio in 2009 (source: https://www.macquarie.com/mgl/ com/news/2009/20090108a.htm).

${ }^{17}$ We also considered other major international markets such as the European and North American markets. However, the trading hours of the Australian market and these international markets do not overlap. As a result, in our network the international markets were strongly linked with the Asian market and had negligible links with all the nodes of Australian economy.

${ }^{18}$ Source: Australian Bureau of Statistics, cat. no. 5209.0.55.001 - Australian National Accounts: Input-
} 


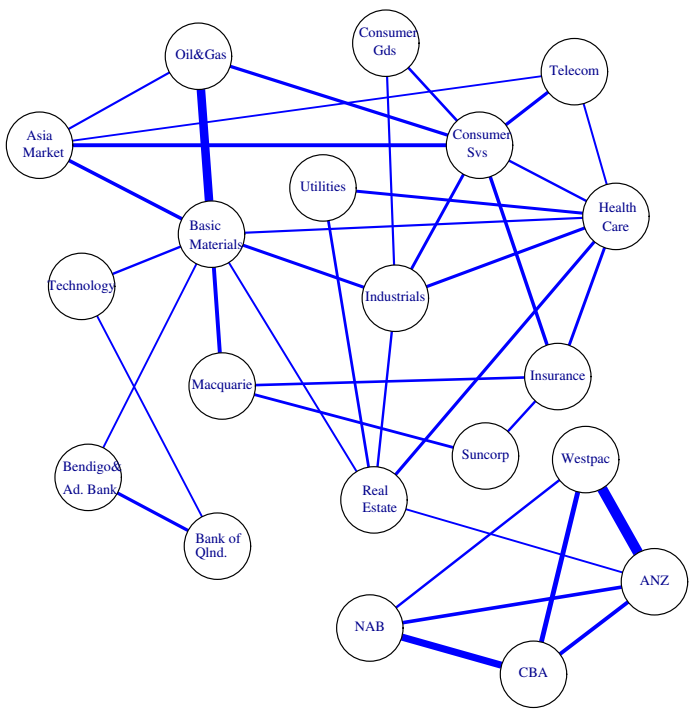

(a) pre-2008

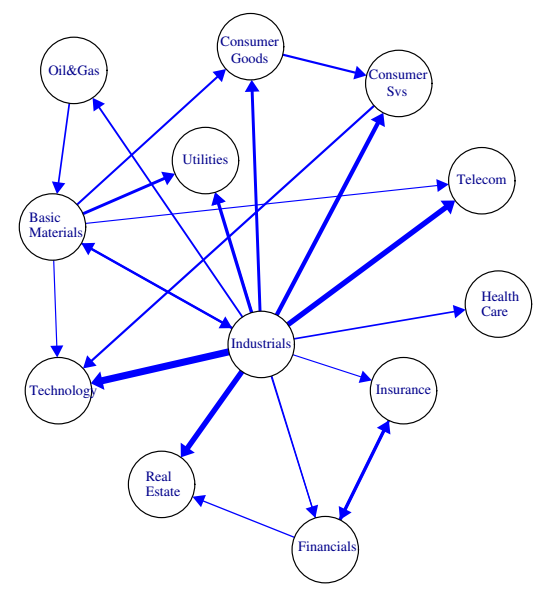

(c) IO $2006-2007$

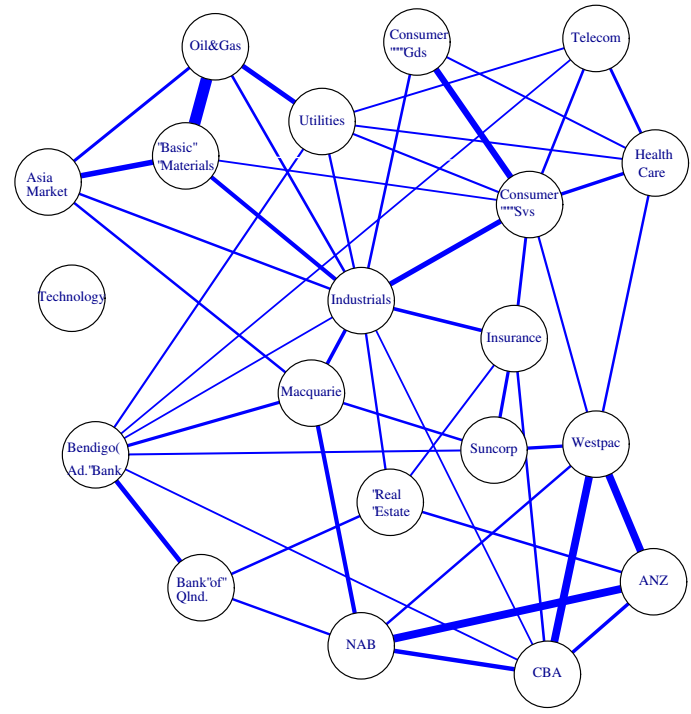

(b) post-2008

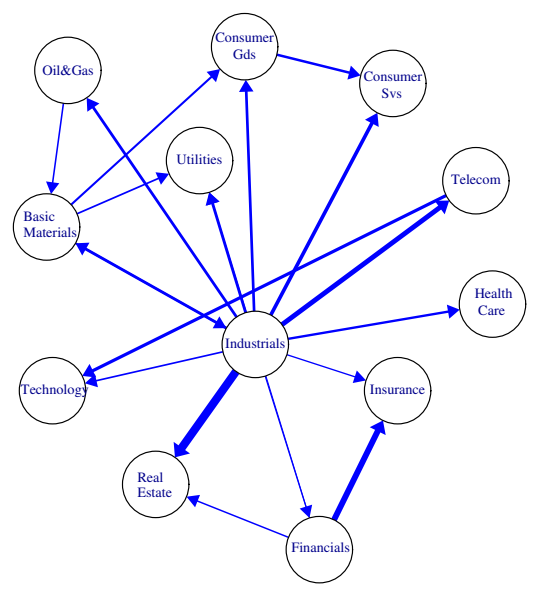

(d) IO $2009-2010$

Figure 2: Evolution of the network of partial correlations (upper panels) and of the Input-Output network (lower panels) of the Australian economy.

post-2008 subsamples of our primary data. The nodes in the networks are sectors of the Australian economy that we matched closely to the Datastream sectoral classification used for the partial correlation network. All banks are included in the Financials sector. The IO network is directed and weighted, with an edge from sector $A$ to sector $B$ carrying the weight equal to the dollar amount of sector $A$ production required to produce a one dollar output of sector $B$. Industrials is at the center of the IO network, as in the network of perceived correlations based on the sector returns. The Financials sector is connected to Output Tables, for the tax years 2006-2007, 2009-2010. 
Table 1: Centrality Measures for the Network of Partial Correlations.

\begin{tabular}{l||c|c|c|c|c|c|c|c|c|c|c|c}
\hline \hline \multicolumn{1}{l||}{} & \multicolumn{2}{|c|}{$\begin{array}{c}R^{2} \\
\text { full }\end{array}$} & \multicolumn{2}{c|}{$\begin{array}{c}\text { Degree } \\
\text { full }\end{array}$} & \multicolumn{2}{c|}{$\begin{array}{c}\text { Eigenvector } \\
\text { full }\end{array}$} & \multicolumn{2}{c|}{$\begin{array}{c}\text { Bonacich } \\
\text { full }\end{array}$} & \multicolumn{2}{c|}{$\begin{array}{c}\text { Bonacich } \\
\text { pre 2008 }\end{array}$} & \multicolumn{2}{c}{$\begin{array}{c}\text { Bonacich } \\
\text { post 2008 }\end{array}$} \\
\hline \hline ANZ & 0.660 & 1 & 1.074 & 4 & 1.000 & 1 & 23.481 & 1 & 13.212 & 2 & 32.294 & 3 \\
Westpac & 0.646 & 2 & 1.056 & 6 & 0.968 & 2 & 22.759 & 3 & 12.454 & 4 & 31.993 & 4 \\
Industrials & 0.621 & 4 & 1.363 & 1 & 0.967 & 3 & 23.478 & 2 & 12.111 & 7 & 40.957 & 1 \\
NAB & 0.618 & 5 & 1.063 & 5 & 0.932 & 4 & 21.993 & 4 & 11.319 & 10 & 31.947 & 5 \\
CBA & 0.609 & 6 & 0.995 & 8 & 0.906 & 5 & 21.333 & 5 & 12.362 & 6 & 29.240 & 6 \\
Basic Materials & 0.623 & 3 & 1.121 & 3 & 0.877 & 6 & 21.189 & 6 & 15.276 & 1 & 28.138 & 8 \\
Consumer Svs & 0.557 & 8 & 1.221 & 2 & 0.831 & 7 & 20.258 & 7 & 11.858 & 8 & 34.707 & 2 \\
Oil \& Gas & 0.578 & 7 & 0.971 & 9 & 0.795 & 8 & 19.204 & 8 & 11.484 & 9 & 28.156 & 7 \\
Insurance & 0.490 & 9 & 1.010 & 7 & 0.743 & 9 & 17.975 & 9 & 12.494 & 3 & 23.976 & 10 \\
Macquarie & 0.469 & 10 & 0.851 & 10 & 0.713 & 10 & 17.031 & 10 & 10.238 & 11 & 23.117 & 11 \\
Asia Market & 0.451 & 11 & 0.793 & 13 & 0.665 & 11 & 15.993 & 11 & 9.161 & 13 & 24.890 & 9 \\
Real Estate & 0.395 & 12 & 0.813 & 11 & 0.615 & 12 & 14.818 & 12 & 9.679 & 12 & 19.196 & 15 \\
Suncorp & 0.377 & 13 & 0.740 & 17 & 0.591 & 13 & 14.135 & 13 & 8.888 & 14 & 18.485 & 16 \\
Bank of Qlnd. & 0.365 & 14 & 0.741 & 16 & 0.570 & 14 & 13.676 & 14 & 7.164 & 15 & 19.419 & 14 \\
Bend\&Ad.Bank & 0.365 & 15 & 0.799 & 12 & 0.564 & 15 & 13.597 & 15 & 6.801 & 16 & 20.748 & 13 \\
Utilities & 0.350 & 17 & 0.766 & 15 & 0.543 & 16 & 13.210 & 16 & 6.680 & 17 & 21.128 & 12 \\
Health Care & 0.351 & 16 & 0.787 & 14 & 0.526 & 17 & 12.874 & 17 & 12.373 & 5 & 14.632 & 18 \\
Consumer Gds & 0.217 & 18 & 0.504 & 18 & 0.363 & 18 & 8.888 & 18 & 6.168 & 18 & 17.513 & 17 \\
Technology & 0.159 & 20 & 0.432 & 20 & 0.306 & 19 & 7.472 & 19 & 4.711 & 20 & 11.205 & 19 \\
Telecom & 0.167 & 19 & 0.464 & 19 & 0.304 & 20 & 7.453 & 20 & 5.472 & 19 & 9.764 & 20 \\
\hline \hline
\end{tabular}

Real estate, Industrials and Insurance. On both networks the Basic Materials sector is linked with Oil \& Gas and Industrials. ${ }^{19}$

In addition to graphical representations of the network of partial correlations, we compute network-based centrality measures which help to identify the most important nodes. Table 1 reports these measures for all economic entities. The first measure is $R^{2}$ as defined in (3), which is the proportion of variation in the returns explained by the returns of all other entities in the network. The remaining measures are the degree centrality, the eigenvector centrality, ${ }^{20}$ and the Bonacich centrality with $\alpha=1$, discussed in Section 3. Next to each measure we report its ranking in the descending order. The rows are ordered according to the eigenvector centrality for the full sample. All measures are reported for the full sample, and for Bonacich centrality we additionally report the values for pre- and

\footnotetext{
${ }^{19}$ The input-output analysis has a long history in economics (see Leontief, 1987). There has been a recent surge of interest in looking at an economy as a linked web of production units (see e.g., Acemoglu et al., 2012; Carvalho, 2014) with an obvious connection to the input-output analysis and the network theory. As our paper is focused on the network of partial correlations, we do not go beyond a simple illustration of the IO network.

${ }^{20}$ The largest absolute eigenvalue is distant from the rest of the eigenvalues. (The three largest absolute eigenvalues are $\lambda_{1}=0.95, \lambda_{2}=0.68$ and $\lambda_{3}=-0.60$.) Therefore, we focus only on the eigenvector centrality as opposed to the $p$-eigenvector centrality space with $p>1$. We normalize the eigenvector corresponding to the largest absolute eigenvalue by fixing its largest component to 1.
} 
post-2008 samples (the other measures for these periods are reported in Appendix D). We notice that the ranking for the full sample is similar for all the considered measures. The eigenvector centrality and the Bonacich centrality measures have the closest ranking similarity with only one different entry.

Using all four measures, ANZ seems to have the most central position. The big four banks together with Industrials have the highest centrality using the full sample. This indicates the importance of the banking sector in shock transmission. Interestingly, Macquarie, which seems to be rather central visually on the full sample and especially post-2008 (Figures 1 and 2b), has moderate centrality measures. This is due to the relatively small weights of its edges, i.e., relatively small partial correlations.

When we compare the pre- and post-2008 periods overall, we notice a significant increase in the network effects. Indeed, the average $R^{2}$ of all entities increases from 0.34 pre-2008 to 0.54 post-2008. Similarly, the average Bonacich centrality increases from 10 to 24. Interestingly, Basic Materials shows the highest centrality pre-2008, and Industrials becomes the most central post-2008. The big four banks remain highly central in both periods. Moreover, their levels of Bonacich centrality increase dramatically post-2008 indicating higher network effects of the banks.

Finally, let us briefly discuss the results based on the Peng et al. (2009) glasso method reported in Appendix E. Due to shrinkage we notice substantially lower values of correlations and somewhat reduced partial correlations in comparison to the baseline case. However, the centrality-based ranking is very similar.

\subsection{Policy examples}

Suppose a policy-maker wishes to lower the total effect of a shock to the economic system in the most effective way. We can measure the total effect of a unit exogenous shock affecting all entities to any specific entity in the system with the Bonacich centrality measure. Using the centrality measures in Table 1, it is easy to identify that ANZ is the most central in the system (based on the full sample).

Let us assume that a policy-maker implements a set of measures reducing the partial correlations of all entities connected with ANZ by 10 percent. We compute that, in this case, the Bonacich centrality measure of ANZ will be reduced by 33 percent from about 23 to 15.3. Moreover, the average Bonacich centrality of all big four banks will be reduced 
by 31 percent from 22.4 to 15.4 , while the average Bonacich centrality of the system will be reduced by 24 percent from 16.5 to 12.5 .

Now, let us check what will happen if a policy-maker were to focus on an important, but less central entity, say the Macquarie group, and reduced its partial correlations by 10 percent. The Bonacich centrality of the Macquarie group would reduce by 23 percent, the average Bonacich centrality of the big four banks would be reduced by 15 percent and the average Bonacich centrality of the whole system would be reduced by 15 percent, which is smaller compared to the optimal policy targeting the most central entity. Focusing on the least central entity such as the Telecom sector and reducing all its connections by 10 percent would reduce the average Bonacich centrality of the system by only 3.5 percent.

It is important to mention that the policy examples considered above are rather stylized and are given merely as an illustration of possible use for the discussed centrality measures. We have only considered the benefits of the policy. However, the costs of reducing the connections of a highly central entity may be substantially higher than the cost of reducing the connections of a less central entity. The centrality measures along with the networks of partial correlations may be used as complementary indicators guiding policy-makers.

\section{Concluding remarks}

In this paper we linked various methods for reconstructing the partial correlation networks, established the connections between the theoretical network measures and principal component analysis, and applied the methodology to reconstruct the implied networks of partial correlations between the relative change in value of the Australian banks, other domestic sectors, and international markets. We investigated the evolution of the networks over time and computed network-based measures for the considered entities.

We found strong direct links between the big four Australian banks and their connection to the real economy via financial services. We demonstrated that the reconstructed network may be somewhat explained by the IO network. A more formal comparison of the perceived partial correlation network and directly observed networks (such as the IO network or the network of interbank exposures) can be an interesting direction for further research. 


\section{References}

Acemoglu, D., Carvalho, V.M., Ozdaglar, A., Tahbaz-Salehi, A., 2012. The network origins of aggregate fluctuations. Econometrica 80, 1977-2016.

Acemoglu, D., Ozdaglar, A., Tahbaz-Salehi, A., 2015. Systemic risk and stability in financial networks. American Economic Review 105, 564-608.

Allen, F., Babus, A., 2009. Networks in finance, in: Kleindorfer, P.R., Wind, Y.J.R., Gunther, R.E. (Eds.), The Network Challenge: Strategy, Profit, and Risk in an Interlinked World.

Ballester, C., Calvó-Armengol, A., Zenou, Y., 2006. Who's who in networks. wanted: the key player. Econometrica 74, 1403-1417.

Bao, Y., Lee, T.H., Saltoglu, B., 2007. Comparing density forecast models. Journal of Forecasting 26, 203.

Barigozzi, M., Brownlees, C., 2013. NETS: Network Estimation for Time Series. Working Paper 723. Barcelona Graduate School of Economics.

Battiston, S., Puliga, M., Kaushik, R., Tasca, P., Caldarelli, G., 2012. Debtrank: Too central to fail? financial networks, the fed and systemic risk. Scientific Reports 2.

Bauwens, L., Laurent, S., Rombouts, J.V., 2006. Multivariate garch models: a survey. Journal of Applied Econometrics 21, 79-109.

Belsey, D.A., Kuh, E., Welsch, R.E., 1980. Regression diagnostics: Identifying influential data and sources of collinearity. John Wiley.

Billio, M., Getmansky, M., Lo, A.W., Pelizzon, L., 2012. Econometric measures of connectedness and systemic risk in the finance and insurance sectors. Journal of Financial Economics 104, 535-559.

Bollerslev, T., 1990. Modelling the coherence in short-run nominal exchange rates: A multivariate generalized arch model. The Review of Economics and Statistics 72, 498505.

Bollerslev, T., Chou, R.Y., Kroner, K.F., 1992. Arch modeling in finance: A review of the theory and empirical evidence. Journal of econometrics 52, 5-59. 
Bonacich, P., 1987. Power and centrality: A family of measures. American journal of sociology, 1170-1182.

Bühlmann, P., Van De Geer, S., 2011. Statistics for High-dimensional Data: Methods, Theory and Applications. Springer.

Carvalho, V.M., 2014. From micro to macro via production networks. The Journal of Economic Perspectives, 23-47.

Chinazzi, M., Fagiolo, G., 2013. Systemic Risk, Contagion, and Financial Networks: A Survey. Technical Report. LEM Working Paper Series.

Creal, D., Koopman, S.J., Lucas, A., 2013. Generalized autoregressive score models with applications. Journal of Applied Econometrics 28, 777-795.

Diebold, F.X., Yilmaz, K., 2014. On the network topology of variance decompositions: Measuring the connectedness of financial firms. Journal of Econometrics 182, 119-134.

Diks, C., Panchenko, V., van Dijk, D., 2010. Out-of-sample comparison of copula specifications in multivariate density forecasts. Journal of Economic Dynamics and Control $34,1596-1609$.

Diks, C., Panchenko, V., van Dijk, D., 2011. Likelihood-based scoring rules for comparing density forecasts in tails. Journal of Econometrics 163, 215-230.

Diks, C., Panchenko, V., Sokolinskiy, O., Van Dijk, D., 2014. Comparing the accuracy of copula-based multivariate density forecasts in selected regions of support. Journal of Economic Dynamics and Control 48, 79-94.

Dungey, M., Luciani, M., Matei, M., Veredas, D., 2015. Surfing through the GFC: Systemic risk in Australia. Working Paper 061. Centre for International Finance and Regulation.

Dungey, M., Luciani, M., Veredas, D., 2013. Googling SIFIs. Working Paper. Centre for International Finance and Regulation.

Elliott, M., Golub, B., Jackson, M.O., 2014. Financial networks and contagion. American Economic Review 104, 3115-3153. 
Engle, R., 2002. Dynamic conditional correlation: A simple class of multivariate generalized autoregressive conditional heteroskedasticity models. Journal of Business \& Economic Statistics 20, 339-350.

Glasserman, P., Young, H.P., 2015. How likely is contagion in financial networks? Journal of Banking and Finance 50, 383-399.

Granger, C.W., 1969. Investigating causal relations by econometric models and crossspectral methods. Econometrica 37, 424-38.

Hastie, T., Tibshirani, R., Friedman, J., 2009. The Elements of Statistical Learning. volume 2. Springer.

Iori, G., De Masi, G., Precup, O.V., Gabbi, G., Caldarelli, G., 2008. A network analysis of the italian overnight money market. Journal of Economic Dynamics and Control 32, $259-278$.

Jackson, M.O., 2008. Social and Economic Networks. Princeton University Press.

Kim, S., Shephard, N., Chib, S., 1998. Stochastic volatility: likelihood inference and comparison with arch models. The Review of Economic Studies 65, 361-393.

Lee, T.H., Long, X., 2009. Copula-based multivariate garch model with uncorrelated dependent errors. Journal of Econometrics 150, 207-218.

Leontief, W., 1987. Input-output analysis. The New Palgrave. A Dictionary of Economics $2,860-64$.

Malioutov, D.M., Johnson, J.K., Willsky, A.S., 2006. Walk-sums and belief propagation in gaussian graphical models. The Journal of Machine Learning Research 7, 2031-2064.

Markowitz, H., 1952. Portfolio selection. The Journal of Finance 7, 77-91.

Meyer, C.D., 2000. Matrix Analysis and Applied Linear Algebra. Siam.

Newman, M., 2010. Networks: An Introduction. Oxford University Press.

Peng, J., Wang, P., Zhou, N., Zhu, J., 2009. Partial correlation estimation by joint sparse regression models. Journal of the American Statistical Association 104. 
Rice, J.J., Tu, Y., Stolovitzky, G., 2005. Reconstructing biological networks using conditional correlation analysis. Bioinformatics 21, 765-773.

Sokolov, A., Webster, R., Melatos, A., Kieu, T., 2012. Loan and nonloan flows in the australian interbank network. Physica A: Statistical Mechanics and its Applications $391,2867-2882$.

Vitali, S., Glattfelder, J.B., Battiston, S., 2011. The network of global corporate control. PloS one 6, e25995.

Whittaker, J., 2009. Graphical Models in Applied Multivariate Statistics. Wiley Publishing. 


\section{APPENDIX}

\section{A Properties of Partial Correlations}

This Appendix collects the results on partial correlations which are used in this paper; most of them are mentioned in Section 2. Many results about partial correlations can be found in Chapter 13 of Bühlmann and Van De Geer (2011), Chapter 17 of Hastie et al. (2009) and Chapter 5 of Whittaker (2009). We selected here the results we need in this paper and adopted them to our notation.

First, we reproduce the definition. Let $X$ be the multivariate random variable with elements $X_{1}, \ldots, X_{n}$. Let $X_{i j \mid}^{*}$. denote the best linear approximation of variable $X_{i}$ based on all the variables except for $X_{i}$ and $X_{j}$ for any pair $i, j$.

Definition. The partial correlation coefficient between $X_{i}$ and $X_{j}$ denoted by $\rho_{i j \mid}$. is defined as the ordinary correlation coefficient between $X_{i}-X_{i j \mid}^{*}$. and $X_{j}-X_{j i \mid}^{*}$.

Without loss of generality we can assume that all the elements of $X$ have zero mean.

For the sake of notation simplicity, the proofs will be focused on random vectors $X_{1}$ and $X_{2}$. We denote $\epsilon_{12}=X_{1}-X_{12}^{*}$. and $\epsilon_{21}=X_{2}-X_{21 \mid}^{*}$. the elements of $X_{1}$ and $X_{2}$ orthogonal to the space spanned by the remaining random variables $X_{3}, \ldots, X_{n}$. By definition the partial correlation coefficient between $X_{1}$ and $X_{2}$ is

$$
\rho_{12 \mid} \cdot=\frac{\mathrm{E}\left(\epsilon_{12} \epsilon_{21}\right)}{\sqrt{\mathrm{E} \epsilon_{12}^{2}} \sqrt{\mathrm{E} \epsilon_{21}^{2}}} .
$$

\section{A.1 Connection with Linear Regression}

To get representation (1) we project $X_{1}$ on all the remaining elements of $X$ and also project $X_{2}$ on all the remaining elements of $X$ (recall that in this appendix the variables are already transformed to have zero mean):

$$
\begin{aligned}
& X_{1}=\beta_{12} X_{2}+\beta_{13} X_{3}+\cdots+\beta_{1 n} X_{n}+\varepsilon_{1} \\
& X_{2}=\beta_{21} X_{1}+\beta_{23} X_{3}+\cdots+\beta_{2 n} X_{n}+\varepsilon_{2} .
\end{aligned}
$$

We will assume that the elements of $X$ are linearly independent and so the projection errors $\varepsilon_{1}$ and $\varepsilon_{2}$ are not constant. The next result establishes the link between $\mathrm{E} \epsilon_{12}^{2}$, the variance of the projection error when $X_{1}$ is projected on the space spanned by $X_{3}, \ldots, X_{n}$, and $\mathrm{E} \varepsilon_{1}^{2}$, the variance of the projection error when $X_{1}$ is projected on the space spanned by $X_{2}, X_{3}, \ldots, X_{n}$. The latter is called the conditional variance of $X_{1}$ in the main text.

Lemma. Assume the orthogonality conditions $\mathrm{E}\left(\varepsilon_{1} X_{i}\right)=0$ for all $i \neq 1$ and $\mathrm{E}\left(\varepsilon_{2} X_{i}\right)=0$ for all $i \neq 2$. Then

$$
\mathrm{E} \epsilon_{12}^{2}=\frac{\mathrm{E} \varepsilon_{1}^{2}}{1-\beta_{12} \beta_{21}} .
$$

Proof. Using several times the orthogonality of $\epsilon_{12}$ to the space spanned by $X_{3}, \ldots, X_{n}$ and substituting $X_{1}$ and $X_{2}$ from (11), we obtain that

$$
\begin{aligned}
\mathrm{E} \epsilon_{12}^{2} & =\mathrm{E}\left(X_{1} \epsilon_{12}\right)=\mathrm{E}\left(\left(\beta_{12} X_{2}+\varepsilon_{1}\right) \epsilon_{12}\right)=\beta_{12} \mathrm{E}\left(X_{2} \epsilon_{12}\right)+\mathrm{E} \varepsilon_{1}^{2}= \\
& =\beta_{12} \mathrm{E}\left(\left(\beta_{21} X_{1}+\varepsilon_{2}\right) \epsilon_{12}\right)+\mathrm{E} \varepsilon_{1}^{2}=\beta_{12} \beta_{21} \mathrm{E} \epsilon_{12}^{2}+\mathrm{E} \varepsilon_{1}^{2} .
\end{aligned}
$$

At the last step we used $\mathrm{E}\left(\varepsilon_{2} \epsilon_{12}\right)=0$ which holds due to the orthogonality conditions for $\varepsilon_{2}$. Note that $\epsilon_{12}=X_{1}-X_{12 \text { | }}^{*}$ is a linear combination of $X_{1}, X_{3}, \ldots, X_{n}$. 
The required result follows now from the equality derived above. Note that $\beta_{12} \beta_{21} \neq 1$, as otherwise $\mathrm{E} \varepsilon_{1}^{2}=0$. But this would contradict the assumption of linear independence of $X_{1}, \ldots, X_{n}$.

Proposition 2. Assume the orthogonality conditions $\mathrm{E}\left(\varepsilon_{1} X_{i}\right)=0$ for all $i \neq 1$ and $\mathrm{E}\left(\varepsilon_{2} X_{i}\right)=0$ for all $i \neq 2$ in (11). Then

$$
\beta_{12}=\rho_{12}\left|\cdot \sqrt{\frac{\mathrm{E} \varepsilon_{1}^{2}}{\mathrm{E} \varepsilon_{1}^{2}}}=\rho_{12}\right| \cdot \sqrt{\frac{\operatorname{Var}\left(\varepsilon_{1}\right)}{\operatorname{Var}\left(\varepsilon_{2}\right)}}
$$

Proof. Let's compute $\mathrm{E}\left(\varepsilon_{1} \epsilon_{21}\right)$. On the one hand, we can express $\varepsilon_{1}$ from (11) and due to orthogonality of $\epsilon_{21}$ to $X_{3}, \ldots, X_{n}$ we get

$$
\mathrm{E}\left(\varepsilon_{1} \epsilon_{21}\right)=\mathrm{E}\left(X_{1} \epsilon_{21}\right)-\beta_{12} \mathrm{E}\left(X_{2} \epsilon_{21}\right) .
$$

On the other hand, $\epsilon_{21}=X_{2}-X_{21 \mid}^{*}$. is a linear combination of $X_{2}, \ldots, X_{n}$ and $\varepsilon_{1}$ is orthogonal to all these vectors. Hence, $\mathrm{E}\left(\varepsilon_{1} \epsilon_{21}\right)=0$ and therefore

$$
\beta_{12}=\frac{\mathrm{E}\left(X_{1} \epsilon_{21}\right)}{\mathrm{E}\left(X_{2} \epsilon_{21}\right)}=\frac{\mathrm{E}\left(\left(X_{12 \mid}^{*}+\epsilon_{12}\right) \epsilon_{21}\right)}{\mathrm{E}\left(\left(X_{21 \mid}^{*}+\epsilon_{21}\right) \epsilon_{21}\right)}=\frac{\mathrm{E}\left(\epsilon_{12} \epsilon_{21}\right)}{\mathrm{E} \epsilon_{21}^{2}}=\rho_{12 \mid} \cdot \sqrt{\frac{\mathrm{E} \epsilon_{12}^{2}}{\mathrm{E} \epsilon_{21}^{2}}},
$$

where at the last step we used the definition of the partial correlation. The previous lemma implies that the ratio of variances of $\epsilon_{12}$ and $\epsilon_{21}$ is the same as the ratio of variances of $\varepsilon_{1}$ and $\varepsilon_{2}$. It completes the proof.

From the last Proposition by symmetry we derive that

$$
\beta_{12} \beta_{21}=\rho_{12 \mid}^{2} . \quad \Leftrightarrow \quad \rho_{12 \mid}=\operatorname{sign}\left(\beta_{12}\right) \sqrt{\beta_{12} \beta_{21}}
$$

Since correlation coefficient is always between -1 and 1 , it follows (see the previous Lemma) that $\beta_{12} \beta_{21}<1$ and that $\mathrm{E} \varepsilon_{1}^{2}<\mathrm{E} \epsilon_{12}^{2}$.

These results hold for any $i$ and $j$ and allow us to establish a link between the matrix of partial correlations, $\mathbf{P}$, and the matrix of linear coefficients in system (1), $\mathbf{B}$. (Both matrices have zeros on the diagonal.) In the main text we defined the diagonal matrix $\mathbf{D}_{\boldsymbol{\Sigma}}=\operatorname{diag}\left\{\operatorname{Var}\left(\varepsilon_{1}\right), \ldots, \operatorname{Var}\left(\varepsilon_{n}\right)\right\}$. The result of Proposition 2 implies that $\mathbf{B}=\mathbf{D}_{\Sigma}^{1 / 2} \mathbf{P} \mathbf{D}_{\Sigma}^{-1 / 2}$. With this result we can directly obtain system (5) which played a crucial role in our separation of the first-order effect of the shock from the total effect of the shock.

\section{A.2 Connection with Concentration Matrix}

To obtain a useful characterization of partial correlation, we investigate the elements of the concentration matrix $\mathbf{K}=\mathbf{\Omega}^{-1}$. Without loss of generality, we will focus only on the first row and the first column of this matrix.

Let $\mathbf{X}$ denote the matrix corresponding to the multivariate random variable $X$. The columns of $\mathbf{X}$ are the random vectors $X_{1}, \ldots, X_{n}$. Let $\mathbf{X}_{-1}$ denote the matrix whose columns are the random vectors $X_{2}, \ldots, X_{n}$. Consider the first equality in (11), and write it as $X_{1}=\mathbf{X}_{-1} \boldsymbol{\beta}+\varepsilon_{1}$, where $\boldsymbol{\beta}=\left(\beta_{12} \cdots \beta_{1 n}\right)^{T}$. Using the orthogonality condition, we obtain from the normal equations that

$$
\boldsymbol{\beta}=\left(\mathrm{E}\left(\mathbf{X}_{-1}^{T} \mathbf{X}_{-1}\right)\right)^{-1} \mathrm{E}\left(\mathbf{X}_{-1}^{T} X_{1}\right) .
$$

The next result exploits the block structure of the variance-covariance matrix of $X$. 
Lemma. Variance-covariance matrix of $\mathbf{X}$ can be represented as follows

$$
\boldsymbol{\Omega}=\mathrm{E}\left(\mathbf{X}^{T} \mathbf{X}\right)=\left(\begin{array}{cc}
1 & \boldsymbol{\beta}^{T} \\
0 & \mathbf{I}
\end{array}\right)\left(\begin{array}{cc}
\operatorname{Var}\left(\varepsilon_{1}\right) & 0 \\
0 & \mathrm{E}\left(\mathbf{X}_{-1}^{T} \mathbf{X}_{-1}\right)
\end{array}\right)\left(\begin{array}{cc}
1 & 0 \\
\boldsymbol{\beta} & \mathbf{I}
\end{array}\right)
$$

Proof. This can be checked by direct computation. For example, variance of $X_{1}$ is

$$
\mathrm{E}\left(X_{1}^{T} X_{1}\right)=\mathrm{E}\left(\left(\boldsymbol{\beta}^{T} \mathbf{X}_{-1}^{T}+\varepsilon_{1}^{T}\right)\left(\mathbf{X}_{-1} \boldsymbol{\beta}+\varepsilon_{1}\right)\right)=\boldsymbol{\beta}^{T} \mathrm{E}\left(\mathbf{X}_{-1}^{T} \mathbf{X}_{-1}\right) \boldsymbol{\beta}+\operatorname{Var}\left(\varepsilon_{1}\right)
$$

which is exactly the upper left element from the right-hand side of the equality. Also, the row vector of covariances of $X_{1}$ with the remaining vectors is

$$
\mathrm{E}\left(X_{1}^{T} \mathbf{X}_{-1}\right)=\mathrm{E}\left(\left(\boldsymbol{\beta}^{T} \mathbf{X}_{-1}^{T}+\varepsilon_{1}^{T}\right) \mathbf{X}_{-1}\right)=\mathrm{E}\left(\boldsymbol{\beta}^{T} \mathbf{X}_{-1}^{T} \mathbf{X}_{-1}\right)
$$

which coincides with the upper right element of the right-hand side.

Using the decomposition of $\boldsymbol{\Omega}$ derived in the previous Lemma we can express the concentration matrix, $\mathbf{K}=\boldsymbol{\Omega}^{-1}$ in the block form as well:

$$
\begin{aligned}
\boldsymbol{\Omega}^{-1} & =\left(\begin{array}{cc}
1 & 0 \\
\boldsymbol{\beta} & \mathbf{I}
\end{array}\right)^{-1}\left(\begin{array}{cc}
\frac{1}{\operatorname{Var}\left(\varepsilon_{1}\right)} & 0 \\
0 & \left(\mathrm{E}\left(\mathbf{X}_{-1}^{T} \mathbf{X}_{-1}\right)\right)^{-1}
\end{array}\right)\left(\begin{array}{cc}
1 & \boldsymbol{\beta}^{T} \\
0 & \mathbf{I}
\end{array}\right)^{-1}= \\
& =\left(\begin{array}{cc}
1 & 0 \\
-\boldsymbol{\beta} & \mathbf{I}
\end{array}\right)\left(\begin{array}{cc}
\frac{1}{\operatorname{Var}\left(\varepsilon_{1}\right)} & 0 \\
0 & \left(\mathrm{E}\left(\mathbf{X}_{-1}^{T} \mathbf{X}_{-1}\right)\right)^{-1}
\end{array}\right)\left(\begin{array}{cc}
1 & -\boldsymbol{\beta}^{T} \\
0 & \mathbf{I}
\end{array}\right)= \\
& =\left(\begin{array}{cc}
\frac{1}{\operatorname{Var}\left(\varepsilon_{1}\right)} & -\frac{1}{\operatorname{Var}\left(\varepsilon_{1}\right)} \boldsymbol{\beta}^{T} \\
-\frac{1}{\operatorname{Var}\left(\varepsilon_{1}\right)} \boldsymbol{\beta} & \frac{1}{\operatorname{Var}\left(\varepsilon_{1}\right)} \boldsymbol{\beta} \boldsymbol{\beta}^{T}+\left(\mathrm{E}\left(\mathbf{X}_{-1}^{T} \mathbf{X}_{-1}\right)\right)^{-1}
\end{array}\right)
\end{aligned}
$$

Studying the elements in the first row of this matrix we obtain the following results for the concentration matrix. The upper left element, $k_{11}=1 / \operatorname{Var}\left(\varepsilon_{1}\right)$. The second element in the first row $k_{12}=-\beta_{12} k_{11}$ which using Proposition 2 can be rewritten as

$$
k_{12}=-\beta_{12} k_{11}=-\rho_{12}\left|\cdot \sqrt{\frac{\operatorname{Var}\left(\varepsilon_{1}\right)}{\operatorname{Var}\left(\varepsilon_{2}\right)}} k_{11}=-\rho_{12}\right| \cdot \sqrt{k_{11} k_{22}} .
$$

Expressing the partial correlation from the equation we obtain an instance of (2). In general, the following holds.

Proposition 3. The on-diagonal elements of the concentration matrix $\mathbf{K}$ are given by

$$
k_{i i}=\frac{1}{\operatorname{Var}\left(\varepsilon_{i}\right)} .
$$

The off-diagonal elements of the concentration matrix $\mathbf{K}$ are proportional to the negative of the corresponding partial correlations. Namely, Eq. (2) holds

$$
\rho_{i j \mid}=\frac{-k_{i j}}{\sqrt{k_{i i} k_{j j}}} .
$$

The last result in the matrix form is Eq. (4).

\section{A.3 Connection with the Inverse of Correlation Matrix}

We use the matrix notation. Let $\mathbf{D}_{\boldsymbol{\Omega}}$ be the diagonal matrix composed of the diagonal elements of $\boldsymbol{\Omega}$. Then, by definition, the correlation matrix of $X$, denoted as $\mathbf{R}$ can be 
written as $\mathbf{R}=\mathbf{D}_{\boldsymbol{\Omega}}^{-1 / 2} \boldsymbol{\Omega} \mathbf{D}_{\boldsymbol{\Omega}}^{-1 / 2}$. Therefore,

$$
\mathbf{R}^{-1}=\mathbf{D}_{\Omega}^{1 / 2} \mathbf{K D}_{\Omega}^{1 / 2}
$$

Thus, $(i, j)$ element of matrix $\mathbf{R}^{-1}$ is $k_{i j} \sqrt{\operatorname{Var}\left(X_{i}\right)} \sqrt{\operatorname{Var}\left(X_{j}\right)}$. With such proportionality to the elements of $\mathbf{K}$, the partial correlations can also be computed if in Eq. (2) the elements of $\mathbf{K}$ are substituted by the elements of $\mathbf{R}^{-1}$. Using (13) we find that the diagonal elements of $\mathbf{R}^{-1}$ are $\operatorname{Var}\left(X_{i}\right) / \operatorname{Var}\left(\varepsilon_{i}\right)$.

\section{A.4 Spectrum of Matrix of Partial Correlations.}

Interpretation of the network of partial correlations given in section 2.1 and discussion in section 3 relies on Assumption 1. This Assumption says that, $\rho(\mathbf{P})$, the spectral radius (i.e., the largest absolute eigenvalue) of matrix $\mathbf{P}$ is less than 1.

It is well known (see, e.g., Meyer, 2000, page 618) that this Assumption is equivalent to each of the following:

- the convergence of a so-called Neumann series,

$$
\mathbf{I}+\mathbf{P}+\mathbf{P}^{2}+\ldots,
$$

which then is equal to $(\mathbf{I}-\mathbf{P})^{-1}$.

- $\lim _{k \rightarrow \infty} \mathbf{P}^{k}=\mathbf{0}$, which is a zero-matrix.

The former fact is used in deriving Eq. (6), and thus this Assumption is crucial for our interpretation. The latter fact is mentioned in footnote 4.

While Assumption 1 is often made in the literature on partial correlations (see e.g., Malioutov et al., 2006), it is important to ask when is this Assumption 1 satisfied. The following general result can be used to derive sufficient conditions.

Proposition 4. For square matrix $\mathbf{A}$ it holds that $\rho(\mathbf{A}) \leq\|\mathbf{A}\|$ for every matrix norm.

The elementary proof of this result can be found in page 497 of Meyer (2000). Since the largest absolute row sum, $\max _{i} \sum_{j}\left|a_{i j}\right|$, is a matrix norm, we can conclude that when all the absolute row sums in $\mathbf{P}$ are less than 1, Assumption 1 is satisfied.

Other sufficient conditions can be found, using the fact that $\mathbf{P}$ is the matrix of partial correlations.

Lemma. All eigenvalues of the matrix of partial correlations are less than 1.

Proof. In Proposition 1 we establish a one-to-one relation between eigenvalues of $\mathbf{P}$ and the eigenvalues of a certain variance-covariance matrix. Namely, if $\lambda$ is the eigenvalue of the former matrix, $1 /(1-\lambda)$ is the eigenvalue of the latter matrix. Since variancecovariance matrices are positive-definite, all their eigenvalues are positive. It then follows that all eigenvalues of $\mathbf{P}$ are less than 1 .

With this Lemma we see that Assumption 1 is equivalent to the fact that all the eigenvalues of $\mathbf{P}$ are larger than -1 , i.e., $\lambda_{i}>-1$ for every $i$. In other words, whenever the smallest eigenvalue of $\mathbf{P}$ is larger than -1 , Assumption 1 holds. Moreover, we can also derive the following result.

Proposition 5. When $\mathbf{P}$ is non-negative matrix (i.e., all partial correlations are nonnegative), its spectral radius is less than 1.

Indeed, for the non-negative matrices the Perron-Frobenius theory can be applied. It contains the result (page 670 in Meyer, 2000) that the spectrum of $\mathbf{P}$ is reached on one of the eigenvalues of $\mathbf{P}$. Therefore, the leading absolute eigenvalue of $\mathbf{P}$ is positive. 


\section{B Industry Classification Benchmark}

We adopt the following sectoral classification from Datastream which is based on the Industry Classification Benchmark:

- Oil \& Gas

- Oil and Gas Producers

- Oil Equipment, Services and Distribution

- Basic Materials

- Chemicals

- Basic Resources including Mining and Industries Metals

- Industrials

- Construction and Materials

- Industrial Goods and Services including transportation and business support

- Consumer Goods

- Food and Beverages

- Personal and Household Goods including Home Construction

- Health Care

- Health Care Equipment and Services

- Pharmaceuticals and Biotechnology

- Consumer Services

- Retail

- Media

- Travel and Leisure

- Telecommunications

- Utilities

- Electricity

- Gas, Water and Multi-Utilities

- Technology

- Software and Computer Services

- Financials

- Banks

- Insurance

- Real estate including real estate investment and services and trusts

- Financial services including financial groups

The Financials sector is considered in more details on the network of partial correlations. In particular, we separately consider Insurance and Real estate sub-sectors. The Banks and Financial services are zoomed in even further and are represented by big-four banks (ANZ, CBA, NAB, Westpac) and two regional banks (Bank of Queensland, and Bendigo and Adelaide Bank), by two large financial groups (Suncorp and Macquarie), respectively. 


\section{Correlations and partial correlations}

Tables 2 and 3 report matrices of correlations and partial correlations, respectively, for the full sample. For better readability, the matrix is divided into several blocks corresponding to correlations and cross-correlations between the banks, other sectors of the Australian economy, and the Asian market.

Table 2: Matrix of correlations.

\begin{tabular}{|c|c|c|c|c|c|c|c|c|c|c|c|c|c|c|c|c|c|c|c|c|}
\hline & $\underset{z}{\stackrel{n}{L}}$ & 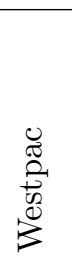 & 爷 & 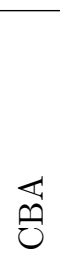 & 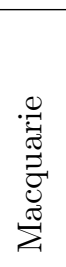 & $\begin{array}{c}\stackrel{0}{0} \\
0 \\
\Xi \\
\Xi \\
\tilde{\Xi}\end{array}$ & 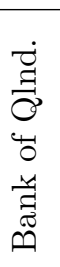 & 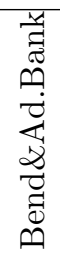 & 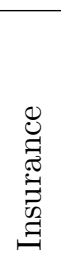 & 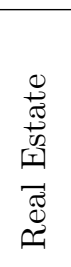 & $\begin{array}{l}\tilde{W} \\
\tilde{W} \\
\infty \\
\bar{\sigma}\end{array}$ & 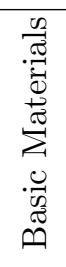 & 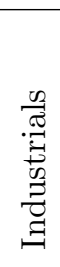 & 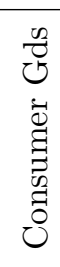 & 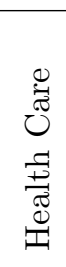 & 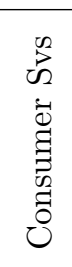 & 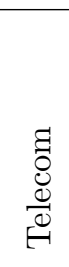 & 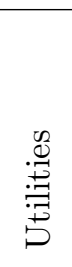 & 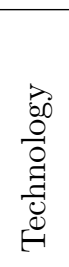 & 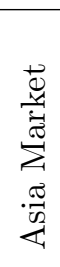 \\
\hline $\mathrm{NAB}$ & 1 & .65 & .71 & .68 & .55 & .47 & .48 & .46 & .49 & .48 & .43 & .44 & .53 & .25 & .36 & .50 & .26 & .39 & .23 & .45 \\
\hline Westpac & .65 & 1 & .73 & .70 & .49 & .49 & .46 & .45 & .49 & .44 & .41 & .44 & .52 & .27 & .40 & .50 & .25 & .40 & .21 & .42 \\
\hline ANZ & .71 & .73 & 1 & .68 & .53 & .47 & .47 & .46 & .50 & .49 & .44 & .45 & .52 & .25 & .36 & .49 & .24 & .38 & .22 & .44 \\
\hline CBA & .68 & .70 & .68 & 1 & .49 & .47 & .45 & .46 & .50 & .45 & .41 & .42 & .54 & .27 & .37 & .49 & .25 & .38 & .23 & .42 \\
\hline Macqu & .55 & .49 & .53 & .49 & 1 & .47 & .42 & .44 & .50 & .45 & .45 & .50 & .55 & .25 & .36 & .47 & .21 & .36 & .25 & .48 \\
\hline Sur & .47 & .49 & .47 & .47 & .47 & 1 & .37 & .40 & .48 & .37 & .39 & .39 & .48 & .27 & .34 & .44 & .22 & .33 & .21 & .38 \\
\hline Ban & .48 & .46 & .47 & .45 & .42 & .37 & 1 & .46 & .42 & .42 & .37 & .39 & .46 & .25 & .31 & .42 & .19 & .34 & .23 & .36 \\
\hline Bend\&Ad.I & .46 & .45 & .46 & .46 & .44 & .40 & .46 & 1 & .44 & .38 & .35 & .39 & .46 & .26 & .31 & .39 & .23 & .36 & .21 & .36 \\
\hline Insur & 49 & .49 & .50 & .50 & .50 & .48 & .42 & .44 & 1 & .46 & .46 & .47 & .58 & .34 & .44 & .57 & .27 & .41 & .25 & .46 \\
\hline Rea & .48 & .44 & .49 & .45 & .45 & .37 & .42 & .38 & .46 & 1 & .39 & .44 & .52 & .27 & .38 & .46 & .24 & .39 & .26 & .40 \\
\hline Oil & .4 & .41 & .44 & .41 & .45 & .39 & .37 & .35 & .46 & .39 & 1 & .72 & .58 & .31 & .39 & .54 & .24 & .45 & .31 & .54 \\
\hline aterials & .44 & .44 & .45 & .42 & .50 & .39 & .39 & .39 & .47 & .44 & .72 & 1 & .63 & .31 & .38 & .53 & .23 & .46 & .34 & .58 \\
\hline Ind & .53 & .52 & .52 & .54 & .55 & .48 & .46 & .46 & .58 & .52 & .58 & .63 & 1 & .39 & .49 & .63 & .29 & .50 & .33 & .53 \\
\hline Gds & .25 & .27 & .25 & .27 & .25 & .27 & .25 & .26 & .34 & .27 & .31 & .31 & .39 & 1 & .31 & .41 & .20 & .28 & .19 & .27 \\
\hline $\mathrm{He}$ & .36 & .40 & .36 & .37 & .36 & .34 & .31 & .31 & .44 & .38 & .39 & .38 & .49 & .31 & 1 & .49 & .30 & .40 & .25 & .34 \\
\hline ner Svs & .50 & .50 & .49 & .49 & .47 & .44 & .42 & .39 & .57 & .46 & .54 & .53 & .63 & .41 & .49 & 1 & .36 & .44 & .30 & .52 \\
\hline Tele & .26 & .25 & .24 & .25 & .21 & .22 & .19 & .23 & .27 & .24 & .24 & .23 & .29 & .20 & .30 & .36 & 1 & .26 & .16 & .26 \\
\hline Utilities & .39 & .40 & .38 & .38 & .36 & .33 & .34 & .36 & .41 & .39 & .45 & .46 & .50 & .28 & .40 & .44 & .26 & 1 & .26 & .37 \\
\hline Technology & .23 & .21 & .22 & .23 & .25 & .21 & .23 & .21 & .25 & .26 & .31 & .34 & .33 & .19 & .25 & .30 & .16 & .26 & 1 & .26 \\
\hline Asia Market & .45 & .42 & .44 & .42 & .48 & .38 & .36 & .36 & .46 & .40 & .54 & .58 & .53 & .27 & .34 & .52 & 2.26 & .37 & .26 & 1 \\
\hline
\end{tabular}


Table 3: Matrix of partial correlations.

\begin{tabular}{|c|c|c|c|c|c|c|c|c|c|c|c|c|c|c|c|c|c|c|c|c|}
\hline & 离 & 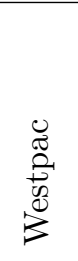 & 爷 & $\stackrel{\leftrightarrow}{\circlearrowright}$ & 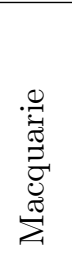 & 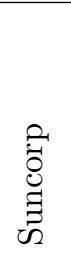 & 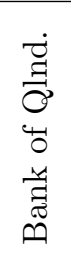 & 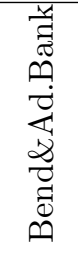 & 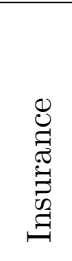 & 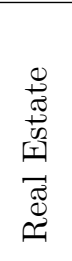 & 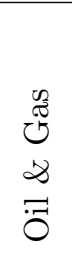 & 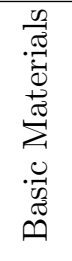 & 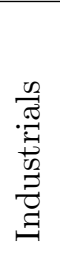 & 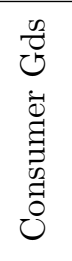 & 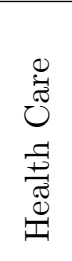 & 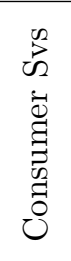 & $\frac{\Xi}{0}$ & 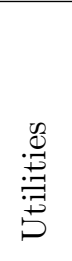 & 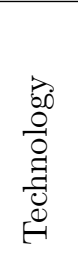 & 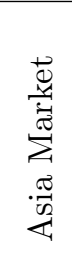 \\
\hline NAB & 0 & .10 & .27 & .24 & .14 & .04 & .08 & .04 & -.01 & .06 & .02 & -.02 & .02 & -.03 & -.01 & .04 & .03 & .02 & .00 & .04 \\
\hline Westpac & .10 & 0 & .37 & .29 & -.01 & .11 & .05 & .02 & .00 & -.03 & -.04 & .05 & .00 & .00 & .08 & .05 & -.01 & .05 & -.04 & .00 \\
\hline $\mathrm{ANZ}$ & .27 & .37 & 0 & .15 & .07 & .01 & .05 & .04 & .04 & .11 & .04 & .00 & .00 & -.02 & -.03 & .00 & -.01 & -.02 & -.01 & .03 \\
\hline $\mathrm{CBA}$ & .24 & .29 & .15 & 0 & .01 & .03 & .02 & .06 & .07 & .01 & .00 & -.03 & .07 & .01 & .00 & .03 & .02 & .01 & .01 & .00 \\
\hline Macquarie & .14 & -.01 & .07 & .01 & 0 & .12 & .04 & .08 & .08 & .07 & .00 & .09 & .10 & -.02 & .02 & .01 & -.04 & -.02 & .01 & .10 \\
\hline Suncorp & .04 & .11 & .01 & .03 & .12 & 0 & .02 & .08 & .12 & .01 & .04 & -.02 & .06 & .04 & .01 & .02 & .02 & .00 & .00 & .01 \\
\hline Bank of Qlnd. & .08 & .05 & .05 & .02 & .04 & .02 & 0 & .18 & .04 & .09 & .01 & .01 & .05 & .02 & -.01 & .04 & -.02 & .02 & .04 & .00 \\
\hline Bend\&Ad.Bank & .04 & .02 & .04 & .06 & .08 & .08 & .18 & 0 & .07 & .02 & -.02 & .02 & .06 & .04 & -.01 & -.04 & .04 & .07 & .01 & .02 \\
\hline Insi & -.01 & .00 & .04 & .07 & .08 & .12 & .04 & .07 & 0 & .07 & .03 & .00 & .10 & .05 & .09 & .15 & .01 & .04 & $\begin{array}{l}.01 \\
\end{array}$ & .06 \\
\hline Real Estate & .06 & -.03 & .11 & .01 & .07 & .01 & .09 & .02 & .07 & 0 & -.03 & .05 & .10 & .01 & .07 & .04 & .03 & .07 & .04 & .03 \\
\hline Oil \& Gas & .02 & -.04 & .04 & .00 & .00 & .04 & .01 & -.02 & .03 & -.03 & 0 & .46 & .07 & .01 & .05 & .09 & .00 & .08 & .03 & .12 \\
\hline Basic Materials & -.02 & .05 & .00 & -.03 & .09 & -.02 & .01 & .02 & .00 & .05 & .46 & 0 & .17 & .02 & -.03 & .03 & -.04 & .09 & .09 & .20 \\
\hline Industrials & .02 & .00 & .00 & .07 & .10 & .06 & .05 & .06 & .10 & .10 & .07 & .17 & 0 & .10 & .10 & .16 & .00 & .10 & .06 & .05 \\
\hline Consumer Gds & -.03 & .00 & -.02 & .01 & -.02 & .04 & .02 & .04 & .05 & .01 & .01 & .02 & .10 & 0 & .07 & .15 & .03 & .04 & .02 & -.01 \\
\hline Health Care & -.01 & .08 & -.03 & .00 & .02 & .01 & -.01 & -.01 & .09 & .07 & .05 & -.03 & .10 & .07 & 0 & .13 & .11 & .11 & .05 & -.02 \\
\hline Consumer Svs & .04 & .05 & .00 & .03 & .01 & .02 & .04 & -.04 & .15 & .04 & .09 & .03 & .16 & .15 & .13 & 0 & .14 & .02 & .04 & .13 \\
\hline Telecom & .03 & -.01 & -.01 & .02 & -.04 & .02 & -.02 & .04 & .01 & .03 & .00 & -.04 & .00 & .03 & .11 & .14 & 0 & .06 & .02 & .05 \\
\hline Utilities & .02 & .05 & -.02 & .01 & -.02 & .00 & .02 & .07 & .04 & .07 & .08 & .09 & .10 & .04 & .11 & .02 & .06 & 0 & .04 & -.01 \\
\hline Technology & .00 & -.04 & -.01 & .01 & .01 & .00 & .04 & .01 & -.01 & .04 & .03 & .09 & .06 & .02 & .05 & .04 & .02 & .04 & 0 & .01 \\
\hline Asia Market & .04 & .00 & .03 & .00 & .10 & .01 & .00 & .02 & .06 & .03 & .12 & .20 & & & -.02 & .13 & .05 & -.01 & .01 & 0 \\
\hline
\end{tabular}




\section{Centrality measures of pre and post 2008 samples}

Tables 4 and 5 show centrality measures of pre and post 2008 samples, respectively. Next to each measure we report the ranking of the corresponding entity according to this measure.

Table 4: Centrality Measures for the Network of Partial Correlations based on pre 2008 sample.

\begin{tabular}{l||c|c|c|c|c|c|c|c}
\hline \hline \multicolumn{1}{l||}{} & \multicolumn{2}{c|}{$R^{2}$} & \multicolumn{2}{c|}{ Degree } & \multicolumn{2}{c|}{ Eigenvector } & \multicolumn{2}{c}{ Bonacich } \\
\hline \hline Basic Materials & 0.553 & 1 & 1.377 & 1 & 1.000 & 1 & 15.276 & 1 \\
ANZ & 0.532 & 2 & 1.015 & 7 & 0.889 & 2 & 13.212 & 2 \\
Westpac & 0.510 & 3 & 0.955 & 8 & 0.839 & 3 & 12.454 & 4 \\
CBA & 0.474 & 4 & 1.041 & 6 & 0.824 & 4 & 12.362 & 6 \\
Insurance & 0.427 & 8 & 1.098 & 3 & 0.819 & 5 & 12.494 & 3 \\
Health Care & 0.431 & 6 & 1.109 & 2 & 0.807 & 6 & 12.373 & 5 \\
Industrials & 0.417 & 10 & 1.068 & 4 & 0.790 & 7 & 12.111 & 7 \\
Consumer Svs & 0.421 & 9 & 1.063 & 5 & 0.776 & 8 & 11.858 & 8 \\
Oil \& Gas & 0.435 & 5 & 0.849 & 11 & 0.761 & 9 & 11.484 & 9 \\
NAB & 0.429 & 7 & 0.888 & 9 & 0.758 & 10 & 11.319 & 10 \\
Macquarie & 0.344 & 11 & 0.811 & 12 & 0.676 & 11 & 10.238 & 11 \\
Real Estate & 0.315 & 12 & 0.857 & 10 & 0.633 & 12 & 9.679 & 12 \\
Asia Market & 0.309 & 13 & 0.712 & 14 & 0.607 & 13 & 9.161 & 13 \\
Suncorp & 0.274 & 14 & 0.784 & 13 & 0.584 & 14 & 8.888 & 14 \\
Bank of Qlnd. & 0.203 & 15 & 0.661 & 15 & 0.467 & 15 & 7.164 & 15 \\
Bend\&Ad.Bank & 0.193 & 17 & 0.639 & 16 & 0.442 & 16 & 6.801 & 16 \\
Utilities & 0.194 & 16 & 0.593 & 17 & 0.435 & 17 & 6.680 & 17 \\
Consumer Gds & 0.172 & 18 & 0.506 & 18 & 0.404 & 18 & 6.168 & 18 \\
Telecom & 0.151 & 19 & 0.505 & 19 & 0.357 & 19 & 5.472 & 19 \\
Technology & 0.111 & 20 & 0.415 & 20 & 0.306 & 20 & 4.711 & 20 \\
\hline \hline
\end{tabular}


Table 5: Centrality Measures for the Network of Partial Correlations based on post 2008 sample.

\begin{tabular}{l||c|c|c|c|c|c|c|c}
\hline \multicolumn{1}{l||}{} & \multicolumn{2}{c|}{$R^{2}$} & \multicolumn{2}{c|}{ Degree } & \multicolumn{2}{c|}{ Eigenvector } & \multicolumn{2}{c}{ Bonacich } \\
\hline \hline Industrials & 0.776 & 1 & 1.618 & 1 & 1.000 & 1 & 40.957 & 1 \\
Consumer Svs & 0.719 & 2 & 1.442 & 2 & 0.844 & 2 & 34.707 & 2 \\
ANZ & 0.714 & 3 & 1.092 & 4 & 0.801 & 3 & 32.294 & 3 \\
Westpac & 0.709 & 4 & 1.059 & 5 & 0.793 & 4 & 31.993 & 4 \\
NAB & 0.702 & 5 & 1.126 & 3 & 0.790 & 5 & 31.947 & 5 \\
CBA & 0.669 & 8 & 0.911 & 7 & 0.725 & 6 & 29.240 & 6 \\
Basic Materials & 0.685 & 6 & 0.886 & 8 & 0.693 & 7 & 28.138 & 8 \\
Oil \& Gas & 0.677 & 7 & 0.992 & 6 & 0.692 & 8 & 28.156 & 7 \\
Asia Market & 0.581 & 9 & 0.848 & 11 & 0.613 & 9 & 24.890 & 9 \\
Insurance & 0.548 & 10 & 0.882 & 9 & 0.587 & 10 & 23.976 & 10 \\
Macquarie & 0.542 & 11 & 0.748 & 13 & 0.571 & 11 & 23.117 & 11 \\
Utilities & 0.502 & 12 & 0.822 & 12 & 0.514 & 12 & 21.128 & 12 \\
Bend.\&Ad.Bank & 0.488 & 13 & 0.855 & 10 & 0.508 & 13 & 20.748 & 13 \\
Bank of Qlnd. & 0.450 & 14 & 0.691 & 15 & 0.477 & 14 & 19.419 & 14 \\
Real Estate & 0.436 & 15 & 0.714 & 14 & 0.470 & 15 & 19.196 & 15 \\
Suncorp & 0.425 & 17 & 0.658 & 16 & 0.455 & 16 & 18.485 & 16 \\
Consumer Gds & 0.433 & 16 & 0.652 & 17 & 0.425 & 17 & 17.513 & 17 \\
Health Care & 0.344 & 18 & 0.602 & 18 & 0.354 & 18 & 14.632 & 18 \\
Technology & 0.235 & 19 & 0.433 & 19 & 0.272 & 19 & 11.205 & 19 \\
Telecom & 0.198 & 20 & 0.421 & 20 & 0.235 & 20 & 9.764 & 20 \\
\hline \hline
\end{tabular}




\section{E Glasso-based estimates and centrality measures}

As a robustness check we estimated the matrices of correlations and partial correlations using the glasso method of Peng et al. (2009). The glasso method exploits the relationship between partial correlations and the system of regression equations in Eq. (1) shrinking the parameters towards zero. The methods relies heavily on the choice of regularization parameter. We use rotation information criterion to choose of the latter. The estimation was implemented in $\mathrm{R}$ using package 'huge'.

Tables 6 and 7 report matrices of correlations and partial correlations, respectively, for the full sample. In addition we also report the centrality measures based on the glasso method in Table 8.

Table 6: Matrix of correlations using glasso method.

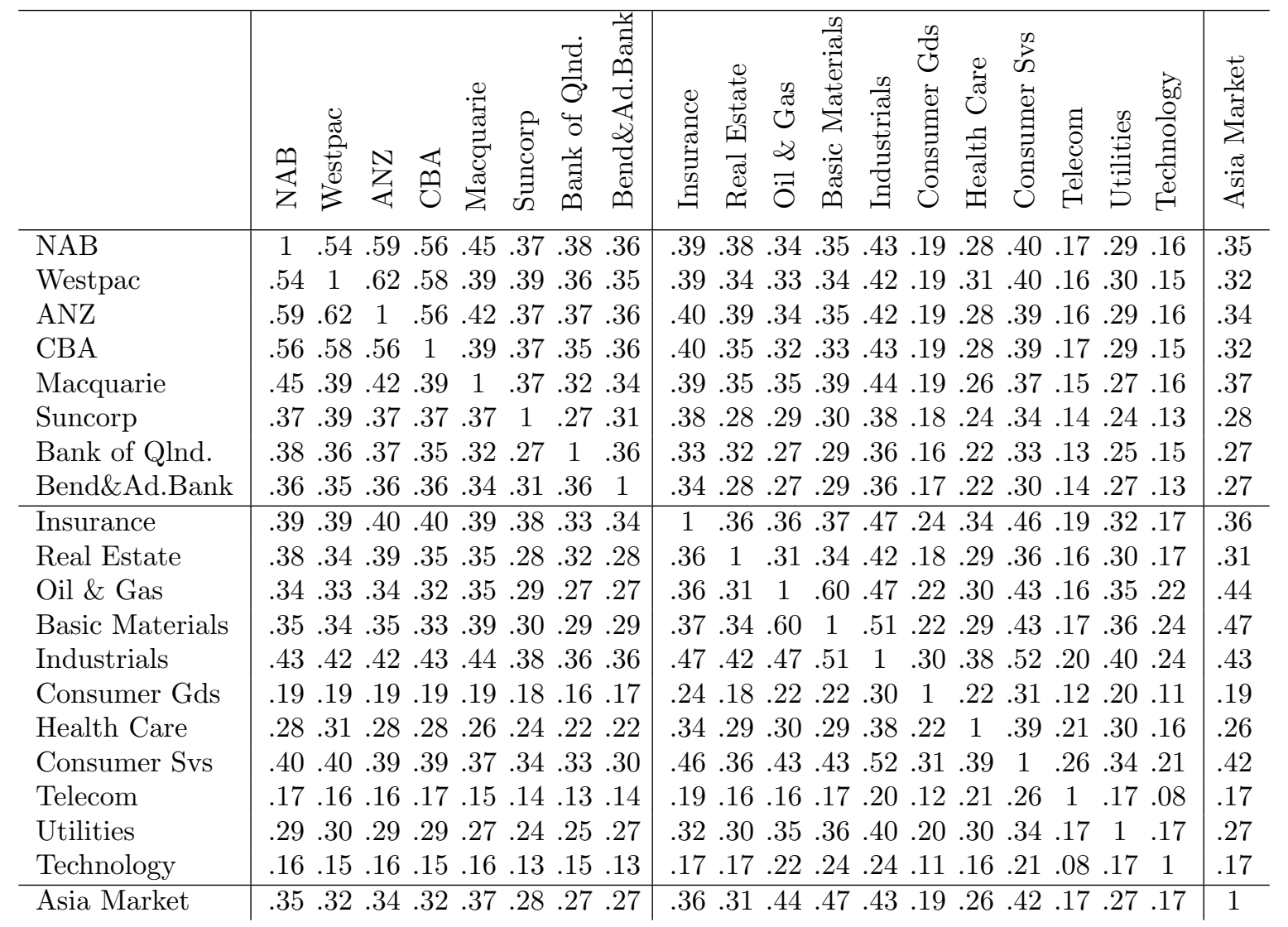


Table 7: Matrix of partial correlations using glasso method.

\begin{tabular}{|c|c|c|c|c|c|c|c|c|c|c|c|c|c|c|c|c|c|c|c|c|}
\hline & 宠 & 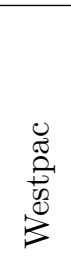 & 爷 & 岇 & 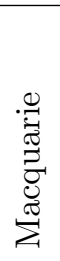 & 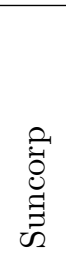 & 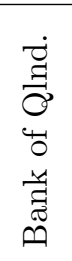 & 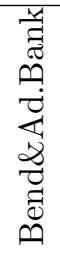 & 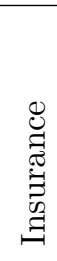 & 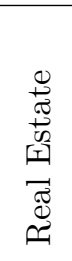 & $\begin{array}{l}\widetilde{D} \\
\tilde{W} \\
\infty \\
\overline{0} \\
\overline{0}\end{array}$ & 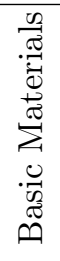 & 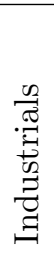 & 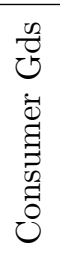 & 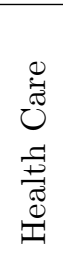 & 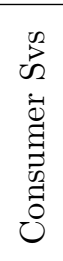 & 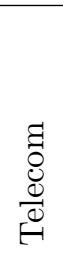 & 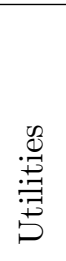 & 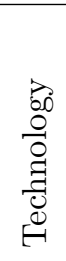 & 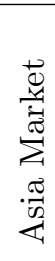 \\
\hline NAB & 0 & .12 & .23 & .20 & .12 & .05 & .08 & .04 & .01 & .06 & .01 & .00 & .02 & 00 & .00 & .04 & .01 & .01 & .00 & .04 \\
\hline Westpac & .12 & 0 & .30 & .25 & .01 & .10 & .05 & .03 & .02 & .00 & .00 & .02 & .02 & .00 & .06 & .05 & .00 & .04 & .00 & .00 \\
\hline ANZ & .23 & .30 & 0 & .15 & .07 & .03 & .05 & .04 & .04 & .09 & .02 & .00 & .01 & .00 & .00 & .00 & .00 & .00 & .00 & .03 \\
\hline $\mathrm{CBA}$ & .20 & .25 & .15 & 0 & .02 & .04 & .03 & .06 & .06 & .02 & .00 & .00 & .06 & .00 & .00 & .03 & .01 & .01 & .00 & .00 \\
\hline Macquarie & .12 & .01 & .07 & .02 & 0 & .10 & .04 & .07 & .07 & .07 & .01 & .08 & .09 & .00 & .00 & .02 & .00 & .00 & .00 & .09 \\
\hline Suncorp & .05 & .10 & .03 & .04 & .10 & 0 & .02 & .07 & .11 & .01 & .03 & .00 & .06 & .02 & .01 & .03 & .00 & .00 & .00 & .02 \\
\hline Bank of Qlnd. & .08 & .05 & .05 & .03 & .04 & .02 & 0 & .15 & .04 & .08 & .01 & .02 & .05 & .00 & .00 & .04 & .00 & .02 & .03 & .01 \\
\hline Bend\&Ad.Bank & .04 & .03 & .04 & .06 & .07 & .07 & .15 & 0 & .06 & .03 & .00 & .02 & .05 & .02 & .00 & .00 & .02 & .06 & .00 & .01 \\
\hline Insurance & .01 & .02 & .04 & .06 & .07 & .11 & .04 & .06 & 0 & .07 & .03 & .01 & .10 & .04 & .09 & .13 & .02 & .04 & .00 & .05 \\
\hline Real Estate & .06 & .00 & .09 & .02 & .07 & .01 & .08 & .03 & .07 & 0 & .00 & .04 & .10 & .00 & .06 & .04 & .01 & .06 & .03 & .03 \\
\hline Oil \& Gas & .01 & .00 & .02 & .00 & .01 & .03 & .01 & .00 & .03 & .00 & 0 & .37 & .08 & .01 & .03 & .09 & .00 & .08 & .04 & .12 \\
\hline Basic Materials & .00 & .02 & .00 & .00 & .08 & .00 & .02 & .02 & .01 & .04 & .37 & 0 & .15 & .02 & .00 & .04 & .00 & .08 & .08 & .18 \\
\hline Industrials & .02 & .02 & .01 & .06 & .09 & .06 & .05 & .05 & .10 & .10 & .08 & .15 & 0 & .09 & .09 & .14 & .01 & .10 & .06 & .06 \\
\hline Consumer Gds & .00 & .00 & .00 & .00 & .00 & .02 & .00 & .02 & .04 & .00 & .01 & .02 & .09 & 0 & .06 & .13 & .01 & .03 & .00 & .00 \\
\hline Health Care & .00 & .06 & .00 & .00 & .00 & .01 & .00 & .00 & .09 & .06 & .03 & .00 & .09 & .06 & 0 & .12 & .09 & .09 & .04 & .00 \\
\hline Consumer Svs & .04 & .05 & .00 & .03 & .02 & .03 & .04 & .00 & .13 & .04 & .09 & .04 & .14 & .13 & .12 & 0 & .12 & .03 & .04 & .11 \\
\hline Telecom & .01 & .00 & .00 & .01 & .00 & .00 & .00 & .02 & .02 & .01 & .00 & .00 & .01 & .01 & .09 & .12 & 0 & .04 & .00 & .03 \\
\hline Utilities & .01 & .04 & .00 & .01 & .00 & .00 & .02 & .06 & .04 & .06 & .08 & .08 & .10 & .03 & .09 & .03 & .04 & 0 & .03 & .00 \\
\hline Technology & .00 & .00 & .00 & .00 & .00 & .00 & .03 & .00 & .00 & .03 & .04 & .08 & .06 & .00 & .04 & .04 & .00 & .03 & 0 & .00 \\
\hline Asia Market & .04 & .00 & .03 & .00 & .09 & .02 & .01 & .01 & .05 & .03 & .12 & .18 & .06 & .00 & .00 & .11 & .03 & .00 & .00 & 0 \\
\hline
\end{tabular}


Table 8: Centrality Measures for the Network of Partial Correlations using glasso method.

\begin{tabular}{l||c|c|c|c|c|c|c|c|c|c|c|c|c}
\hline \hline \multicolumn{1}{l||}{} & \multicolumn{2}{|c|}{$\begin{array}{c}R^{2} \\
\text { full }\end{array}$} & \multicolumn{2}{c|}{$\begin{array}{c}\text { Degree } \\
\text { full }\end{array}$} & \multicolumn{2}{c|}{$\begin{array}{c}\text { Eigenvector } \\
\text { full }\end{array}$} & \multicolumn{2}{c|}{$\begin{array}{c}\text { Bonacich } \\
\text { full }\end{array}$} & \multicolumn{2}{c}{$\begin{array}{c}\text { Bonacich } \\
\text { pre 2008 }\end{array}$} & \multicolumn{2}{c}{$\begin{array}{c}\text { Bonacich } \\
\text { post 2008 }\end{array}$} \\
\hline \hline Industrials & 0.496 & 3 & 1.322 & 1 & 1.000 & 1 & 14.478 & 1 & 7.953 & 5 & 21.904 & 1 \\
ANZ & 0.518 & 1 & 1.063 & 4 & 0.993 & 2 & 13.900 & 2 & 8.335 & 2 & 17.743 & 4 \\
Westpac & 0.504 & 2 & 1.034 & 6 & 0.963 & 3 & 13.490 & 3 & 7.789 & 7 & 17.587 & 5 \\
NAB & 0.486 & 4 & 1.042 & 5 & 0.949 & 4 & 13.329 & 4 & 7.264 & 10 & 17.795 & 3 \\
CBA & 0.476 & 6 & 0.966 & 8 & 0.922 & 5 & 12.907 & 5 & 7.878 & 6 & 16.549 & 6 \\
Basic Materials & 0.479 & 5 & 1.083 & 3 & 0.876 & 6 & 12.622 & 6 & 9.864 & 1 & 15.523 & 7 \\
Consumer Svs & 0.434 & 8 & 1.191 & 2 & 0.868 & 7 & 12.617 & 7 & 7.746 & 8 & 19.306 & 2 \\
Oil \& Gas & 0.437 & 7 & 0.938 & 9 & 0.804 & 8 & 11.546 & 8 & 7.394 & 9 & 15.466 & 8 \\
Insurance & 0.375 & 9 & 0.986 & 7 & 0.787 & 9 & 11.317 & 9 & 8.219 & 3 & 14.250 & 10 \\
Macquarie & 0.355 & 10 & 0.871 & 10 & 0.756 & 10 & 10.744 & 10 & 6.689 & 11 & 13.730 & 11 \\
Asia Market & 0.338 & 11 & 0.783 & 11 & 0.698 & 11 & 9.978 & 11 & 5.895 & 13 & 14.558 & 9 \\
Real Estate & 0.289 & 12 & 0.781 & 12 & 0.652 & 12 & 9.328 & 12 & 6.264 & 12 & 11.500 & 15 \\
Suncorp & 0.275 & 13 & 0.709 & 17 & 0.624 & 13 & 8.862 & 13 & 5.689 & 14 & 11.105 & 16 \\
Bank of Qlnd. & 0.264 & 14 & 0.714 & 16 & 0.601 & 14 & 8.563 & 14 & 4.434 & 15 & 11.677 & 14 \\
Bend\&Ad.Bank & 0.262 & 15 & 0.740 & 14 & 0.595 & 15 & 8.509 & 15 & 4.174 & 16 & 12.449 & 13 \\
Utilities & 0.249 & 16 & 0.733 & 15 & 0.570 & 16 & 8.250 & 16 & 4.102 & 17 & 12.544 & 12 \\
Health Care & 0.247 & 17 & 0.742 & 13 & 0.552 & 17 & 8.035 & 17 & 8.105 & 4 & 8.754 & 18 \\
Consumer Gds & 0.135 & 18 & 0.440 & 18 & 0.371 & 18 & 5.357 & 18 & 3.762 & 18 & 10.414 & 17 \\
Technology & 0.090 & 20 & 0.352 & 20 & 0.299 & 19 & 4.310 & 19 & 2.611 & 20 & 6.653 & 19 \\
Telecom & 0.094 & 19 & 0.380 & 19 & 0.295 & 20 & 4.283 & 20 & 3.232 & 19 & 5.582 & 20 \\
\hline \hline
\end{tabular}

